\title{
Preparation of Carbamates, Esters, Amides, and Unsymmetrical Ureas via Brønsted Acid-Activated $N$-Acyl Imidazoliums
}

Rebecca B. Watson, ${ }^{*}$ Todd W. Butler, Jacob C. DeForest,$*$ *

Chemical Research \& Development, Pharmaceutical Science Small Molecules Division, Worldwide Research and Development, Pfizer Inc., Groton, Connecticut, 06340, United States

\section{Supporting Information}

1. General Methods

2

2. Synthesis of Products

3. NMR Spectra

4. References 


\section{General Methods}

All commercially available chemicals, reagents and solvents were used as received. Reactions were monitored by UPLC-MS (Waters Acquity, ESCI (ESI +/-, APCI +/-)) or by thin layer chromatography (TLC) performed on Analtech, Inc. silica gel GF $250 \mu \mathrm{m}$ plates or Merck silica gel plates (60 F254) and were visualized with ultraviolet (UV) light (254 nm) and/or $\mathrm{KMnO}_{4}$ staining. Proton nuclear magnetic resonance ( ${ }^{1} \mathrm{H}$ NMR) spectra, carbon nuclear magnetic resonance $\left({ }^{13} \mathrm{C} \mathrm{NMR}\right)$ spectra and fluorine nuclear magnetic resonance $\left({ }^{19} \mathrm{~F}\right.$ NMR) spectra were recorded on Bruker or JEOL 400 NMR spectrometers (400, 101 and $376 \mathrm{MHz}$, respectively) equipped with cryoprobes. Chemical shifts are reported in ppm relative to chloroform $\left({ }^{1} \mathrm{H}, \delta=7.26\right.$ and ${ }^{13} \mathrm{C}$ NMR $\left.\delta=77.16\right)$ or dimethyl sulfoxide $\left({ }^{1} \mathrm{H}, \delta=2.50 \mathrm{ppm},{ }^{13} \mathrm{C}\right.$ NMR $\left.\delta=39.52\right)$. The NMR peak multiplicities are denoted as follows: s, singlet; br s, broadened singlet; d, doublet; $\mathrm{t}$, triplet; q, quartet; m, multiplet. Infrared (IR) spectra were recorded with a Nicolet i310 FT-IR. Highresolution mass spectra (HRMS) were acquired on an Agilent model 6220 MS(TOF). UPLC-MS (Waters Acquity), Column: Waters Acquity HSS T3, $2.1 \mathrm{~mm}$ x $50 \mathrm{~mm}, \mathrm{C} 18,1.7 \mu \mathrm{m}$; Column Temperature $60{ }^{\circ} \mathrm{C}$, Mobile Phase: A: $0.1 \%$ formic acid in water (v/v); Mobile phase B: $0.1 \%$ formic acid in acetonitrile (v/v), Gradient Profiles: Flow - $1.25 \mathrm{~mL} / \mathrm{min}, 1.5 \mathrm{~min}$ Run: Initial conditions: A-95\%:B-5\%; hold at initial from 0.0-0.1 min; Linear Ramp to A-5\%:B-95\% over 0.1$1.0 \mathrm{~min}$; hold at A-5\%:B-95\% from 1.0-1.1 min; return to initial conditions 1.1-1.5 min, $3.0 \mathrm{~min}$ run: Initial conditions: A-95\%:B-5\%; hold at initial from 0.0-0.1 min; Linear Ramp to A-5\%:B95\% over 0.1-2.6 min; hold at A-5\%:B-95\% from 2.6-2.95 min; return to initial conditions $2.95-$ 3.0 min. Detectors: Waters Acquity PDA ; 200-450 nm scan; $1.2 \mathrm{~nm}$ interval, Waters Acquity ELS detector; drift tube $65^{\circ} \mathrm{C}$, Waters SQ MS(single quad) Tune: ESI-3.5kV Capillary/APCI (in ESCI mode)- $0.3 \mu \mathrm{A}$ Corona Pin, $30 \mathrm{~V}$ Cone, Source $150{ }^{\circ} \mathrm{C}$, Desolvation $475^{\circ} \mathrm{C}$, Desolvation Gas $\mathrm{N}_{2}$ 400 L/hr, MS Methods: ESCI (ESI+/-, APCI+/-), 100-2000 m/z scan, $0.4 \mathrm{sec}$ scan time, Centroid, Injection Volume: $5 \mu \mathrm{L}$, System Components: Waters Acquity UPLC (Acquity Binary Solvent Manager, 2777C-Autosampler, Acquity PDA, Acquity ELS and Acquity Column Manager) and Waters Acquity SQ systems from Waters Corporation, Milford, MA. Column chromatography was performed on an ISCO Combiflash Rf + system using a pre-packed column or using Baker 40 $\mu \mathrm{M}$ flash chromatography packing silica gel. 


\section{Synthesis of Products}

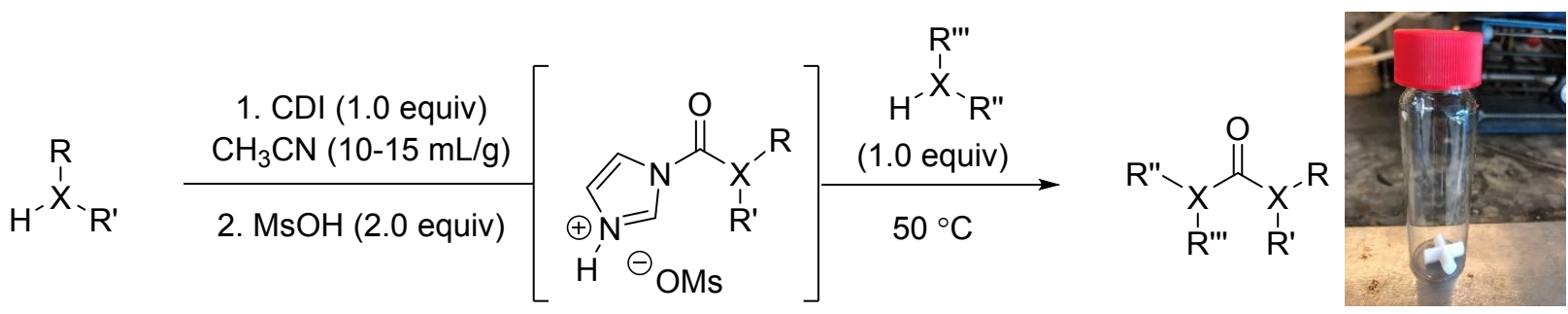

General procedure for the synthesis of amides and esters:

To a $40 \mathrm{~mL}$ vial equipped with a X-shaped magnetic stir bar (see above) and temperature probe, was charged respective carboxylic acid (1.0 equiv) and acetonitrile (10-15 $\left.\mathrm{V}^{\mathrm{i}}\right)$. Stirring was set to 700-1000 rpm. Next, 1,1'-carbonyldiimidazole (CDI) (1.0 equiv) was addedii and the reaction was stirred for 15-30 minutes at room temperature. Methanesulfonic acid (MsOH) (2.0 equiv) was added dropwise over 15-30 minutes with vigorous stirring ${ }^{\text {iii }}$, ensuring the internal temperature of the reaction was kept below $30^{\circ} \mathrm{C}$. The respective phenol or amine (1.0 equiv) was added and the reaction was heated in a pie-block to $50{ }^{\circ} \mathrm{C}$. The resulting mixture was stirred at $50{ }^{\circ} \mathrm{C}$ until judged complete by UPLC analysis. The reaction was cooled to room temperature and subjected to the general workup protocol.

\section{General procedure for the synthesis of carbamates:}

To a $40 \mathrm{~mL}$ vial equipped with a X-shaped magnetic stir bar (see above) and temperature probe, was charged respective aryl alcohol (1.0 equiv) and acetonitrile (10-15 Vi). Stirring was set to 700$1000 \mathrm{rpm}$. Next, 1,1'-carbonyldiimidazole (CDI) (1.0 equiv) was added and the reaction was stirred for 15-30 minutes at room temperature ${ }^{\mathrm{ii}}$. Methanesulfonic acid $(\mathrm{MsOH})$ (2.0 equiv) was added dropwise over 15-30 minutes with vigorous stirringiii, ensuring the internal temperature of the reaction was kept below $30^{\circ} \mathrm{C}$. The respective amine (1.0 equiv) was added and the reaction was heated in a pie-block to $50{ }^{\circ} \mathrm{C}$. The resulting mixture was stirred at $50{ }^{\circ} \mathrm{C}$ until judged complete by UPLC analysis. The reaction was cooled to room temperature and subjected to the general workup protocol.

\section{General procedure for the synthesis of unsymmetrical ureas:}

To a $40 \mathrm{~mL}$ vial equipped with a X-shaped magnetic stir bar (see above) and temperature probe, was charged 1,1'-carbonyldiimidazole (CDI) (1.0 equiv) and acetonitrile (10-15 V'). Stirring was set to 700-1000 rpm. Next, the less nucleophilic amine (1.0 equiv) was added dropwise or portionwise over 15-30 minutes, and the resulting mixture was stirred for 30 minutes at room temperature. Methanesulfonic acid $(\mathrm{MsOH})$ (2.0 equiv) was added dropwise over 15-30 minutes with vigorous stirring iii, ensuring the internal temperature of the reaction was kept below $30{ }^{\circ} \mathrm{C}$. The respective amine (1.0 equiv) was added and the reaction was heated in a pie-block to $50{ }^{\circ} \mathrm{C}$.

${ }^{\mathrm{i}} 1 \mathrm{~V}=1 \mathrm{~mL} / \mathrm{g}$.

ii Gas evolution occurs.

iii Note: If $\mathrm{MsOH}$ is added too fast, the reaction will solidify. If this occurs, addition of $5 \mathrm{~V}$ of acetonitrile will result in a mobile slurry. 
The resulting mixture was stirred at $50{ }^{\circ} \mathrm{C}$ until judged complete by UPLC analysis. The reaction was cooled to room temperature and subjected to the general workup protocol.

\section{General workup:}

After reaching room temperature, the reaction contents were transferred to a larger vessel with water $(80 \mathrm{~V}) .{ }^{\text {iv }}$ If solids precipitated, the mixture was stirred for 30 minutes and the solids were collected by filtration, rinsing with water $(20 \mathrm{~V})$. If no solids precipitated or oiling occurred, the reaction mixture was extracted with EtOAc $(2 \times 30 \mathrm{~mL})$, washed with brine $(30 \mathrm{~mL})$, dried over sodium sulfate, concentrated, and purified by column chromatography.

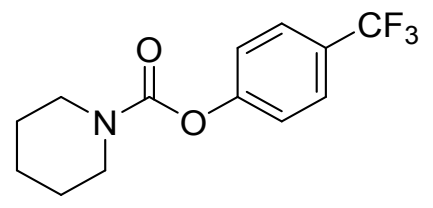

15

4-(Trifluoromethyl)phenyl piperidine-1-carboxylate (15): The title reaction was performed on $1 \mathrm{~g}$ scale with a total reaction time of 1 hour. 4-(Trifluoromethyl)phenol was activated with CDI in $10 \mathrm{~V}$ acetonitrile prior to the addition of $\mathrm{MsOH}$. Isolation via crystallization from water provided $1.21 \mathrm{~g}$ (74\% yield) of 15 as a white solid. ${ }^{1} \mathbf{H}$ NMR (400 MHz; DMSO- $\left.d_{6}\right) \delta 7.76(\mathrm{~d}, J=8.6 \mathrm{~Hz}$, 2H), $7.36(\mathrm{~d}, J=8.5 \mathrm{~Hz}, 2 \mathrm{H}), 3.62-3.50(\mathrm{~m}, 2 \mathrm{H}), 3.49-3.37(\mathrm{~m}, 2 \mathrm{H}), 1.69-1.47(\mathrm{~m}, 6 \mathrm{H}) ;{ }^{13} \mathrm{C}$ NMR $\left(100 \mathrm{MHz} ; \mathrm{CDCl}_{3}\right) \delta 154.3,153.1,127.4(\mathrm{q}, J=32.6 \mathrm{~Hz}), 126.7(\mathrm{q}, J=3.7 \mathrm{~Hz}), 124.2(\mathrm{q}, J$ $=271.8 \mathrm{~Hz}), 122.2$, 45.7, 45.3, 26.0, 25.6, 24.3; ${ }^{19} \mathbf{F}$ NMR $\left(375 \mathrm{MHz} ; \mathrm{CDCl}_{3}\right) \delta$-60.5; IR (Neat) 2951, 2865, 1711, 1612, 1597, 1467, 1429, 1373, 1355, 1321, 1287, 1266, 1233, 1207, 1153, 1143, 1110, 1100, 1063, 1047, 1021, 1012, 979, 957, 906, 875, 853, 831, 797, 746, 729, 657, 634, 592, 533; HRMS (Q-TOF) m/z: [M+H] ${ }^{+}$Calcd for $\mathrm{C}_{13} \mathrm{H}_{15} \mathrm{~F}_{3} \mathrm{NO}_{2}$ : 274.1049; found: 274.1049.<smiles>O=C(Oc1cnc(C(F)(F)F)nc1)N1CCCCC1</smiles>

17

2-(Trifluoromethyl)pyrimidin-5-yl piperidine-1-carboxylate (17): The title reaction was performed on $1 \mathrm{~g}$ scale with a total reaction time of 3 hours. 2-(Trifluoromethyl)-5-pyrimidinol was activated with $\mathrm{CDI}$ in $15 \mathrm{~V}$ acetonitrile prior to the addition of $\mathrm{MsOH}$. Isolation via crystallization from water provided $1.32 \mathrm{~g}\left(60 \%\right.$ yield) of $\mathbf{1 7}$ as a white solid. ${ }^{1} \mathbf{H}$ NMR (400 MHz; DMSO- $\left.d_{6}\right) \delta 9.02(\mathrm{~s}, 2 \mathrm{H}), 3.65-3.56(\mathrm{~m}, 2 \mathrm{H}), 3.48-3.42(\mathrm{~m}, 2 \mathrm{H}), 1.65-1.52(\mathrm{~m}, 6 \mathrm{H}) ;{ }^{13} \mathbf{C ~ N M R}$ $\left(100 \mathrm{MHz}\right.$; DMSO- $\left.d_{6}\right) \delta 151.8,151.0,150.8$ (q, $\left.J=36.1 \mathrm{~Hz}\right), 147.9,119.5$ (q, $\left.J=274.9 \mathrm{~Hz}\right), 45.3$, 44.9, 25.2, 24.9, 23.4; ${ }^{19}$ F NMR (375 MHz; $\left.\mathrm{CDCl}_{3}\right) \delta$-68.3; IR (Neat) 2953, 2928, 2859, 1719 , 1573, 1469, 1453, 1429, 1344, 1284, 1259, 1228, 1183, 1137, 1110, 1045, 1015, 974, 954, 941, 902, 839, 853, 812, 797, 768, 742, 677, 640, 623; HRMS (Q-TOF) m/z: $[\mathrm{M}+\mathrm{H}]^{+}$Calcd for $\mathrm{C}_{11} \mathrm{H}_{13} \mathrm{~F}_{3} \mathrm{~N}_{3} \mathrm{O}_{2}$ : 276.0954; found: 276.0955 .

ivThe $1 H$-imidazol-3-ium methanesulfonate generated during the reaction dissolves in water. 


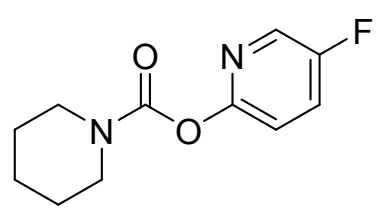

18

5-Fluoropyridin-2-yl piperidine-1-carboxylate (18): The title reaction was performed on $1 \mathrm{~g}$ scale with a total reaction time of 3 hours. 5-Fluoro-2-hydroxypyridine was activated with CDI in $15 \mathrm{~V}$ acetonitrile prior to the addition of $\mathrm{MsOH}$. Purification by flash column chromatography eluting with $n$-heptane/EtOAc provided 18 as a clear oil. The yield of 18 (72\%) was determined by ${ }^{1} \mathrm{H}$ NMR analysis using mesitylene as an internal standard. ${ }^{1} \mathbf{H} \mathbf{~ N M R}\left(400 \mathrm{MHz} ; \mathrm{CDCl}_{3}\right) \delta 8.17$ $(\mathrm{d}, J=3.1 \mathrm{~Hz}, 1 \mathrm{H}), 7.45(\mathrm{ddd}, J=8.9,7.3,3.1 \mathrm{~Hz}, 1 \mathrm{H}), 7.08(\mathrm{dd}, J=8.9,3.7 \mathrm{~Hz}, 1 \mathrm{H}), 3.65-3.54$ $(\mathrm{m}, 2 \mathrm{H}), 3.53-3.45(\mathrm{~m}, 2 \mathrm{H}), 1.66-1.55(\mathrm{~m}, 6 \mathrm{H}) ;{ }^{13} \mathbf{C ~ N M R}\left(100 \mathrm{MHz} ; \mathrm{CDCl}_{3}\right) \delta 157.7(\mathrm{~d}, J=252.3$ $\mathrm{Hz}), 154.6(\mathrm{~d}, J=2.1 \mathrm{~Hz}), 152.8,135.5(\mathrm{~d}, J=26.5 \mathrm{~Hz}), 126.4(\mathrm{~d}, J=20.9 \mathrm{~Hz}), 117.4(\mathrm{~d}, J=5.1$ $\mathrm{Hz}), 45.8,45.2,25.9,25.5,24.3 ;{ }^{19} \mathbf{F}$ NMR $\left(375 \mathrm{MHz} ; \mathrm{CDCl}_{3}\right) \delta$-132.0; IR (Neat) 2938, 2857, 1714, 1599, 1483, 1466, 1422, 1384, 1352, 1237, 1196, 1138, 1051, 1019, 978, 955, 905, 854, 834, 809, 778, 746, 638, 561, 539; HRMS (Q-TOF) m/z: $[\mathrm{M}+\mathrm{H}]^{+}$Calcd for $\mathrm{C}_{11} \mathrm{H}_{14} \mathrm{FN}_{2} \mathrm{O}_{2}$ : 225.1034; found: 225.1031.<smiles>O=C(Oc1c(F)c(F)c(F)c(F)c1F)N1CCCCC1</smiles>

19

Pentafluorophenyl piperidine-1-carboxylate (19): The title reaction was performed on $1 \mathrm{~g}$ scale with a total reaction time of 3 hours. Pentafluorophenol was activated with CDI in $15 \mathrm{~V}$ acetonitrile prior to the addition of $\mathrm{MsOH}$. Purification by flash column chromatography eluting with $n$ heptane/EtOAc provided $0.88 \mathrm{~g}(55 \%$ yield $)$ of 19 as a clear oil. ${ }^{1} \mathbf{H}$ NMR $\left(400 \mathrm{MHz} ; \mathrm{CDCl}_{3}\right) \delta$ 3.69-3.58 (m, 2H), 3.57-3.46 (m, 2H), 1.72-1.61 (m, 6H); ${ }^{19} \mathbf{F}$ NMR (375 MHz; $\left.\mathrm{CDCl}_{3}\right) \delta-153.23$ $--154.47(\mathrm{~m}),-159.45(\mathrm{t}, J=21.7 \mathrm{~Hz}),-163.27$ (td, $J=22.2,4.9 \mathrm{~Hz})$; IR (Neat) 2945, 2863, 1743, $1655,1516,1469,1424,1353,1285,1250,1227,1137,1042,1015,995,955,899,844,820,743$, 717, 635, 607, 539; HRMS (Q-TOF) $\mathbf{~ m / z : ~}[\mathrm{M}+\mathrm{H}]^{+}$Calcd for $\mathrm{C}_{12} \mathrm{H}_{11} \mathrm{~F}_{5} \mathrm{NO}_{2}$ : 296.0704; found: 296.0702 .<smiles>CC(C)(C)OC(=O)N1CCN(C(=O)Oc2cnc(C(F)(F)F)nc2)CC1</smiles>

1-(tert-Butyl) 4-(2-(trifluoromethyl)pyrimidin-5-yl) piperazine-1,4-dicarboxylate (20): The title reaction was performed on $1 \mathrm{~g}$ scale with a total reaction time of 3 hours. 2-(Trifluoromethyl)5-pyrimidinol was activated with CDI in $15 \mathrm{~V}$ acetonitrile prior to the addition of $\mathrm{MsOH}$. Purification by flash column chromatography eluting with $n$-heptane/EtOAc provided $1.93 \mathrm{~g} \mathrm{(88 \%}$ yield) of 20 as a white solid. ${ }^{1} \mathbf{H}$ NMR (400 MHz; DMSO- $\left.d_{6}\right) \delta 9.04(\mathrm{~s}, 2 \mathrm{H}), 3.67-3.60(\mathrm{~m}, 2 \mathrm{H})$, 3.52-3.40 (m, 6H), 1.43 (s, 9H); ${ }^{13} \mathbf{C}$ NMR (100 MHz; DMSO- $\left.d_{6}\right) \delta 153.7,151.8,151.1,151.0(\mathrm{q}$, $J=36.4 \mathrm{~Hz}), 147.7,119.4(\mathrm{q}, J=274.8 \mathrm{~Hz}), 79.3,44.2,43.7,28.0 ;{ }^{19} \mathbf{F}$ NMR $\left(375 \mathrm{MHz} ; \mathrm{CDCl}_{3}\right)$ 
$\delta$-68.3; IR (Neat) 2862,1738, 1684, 1570, 1480, 1457, 1421, 1393, 1367, 1350, 1288, 1225, 1184 , $1165,1141,1115,1080,1043,997,952,942,861,841,829,817,800,769,741,680,644,629$, 578, 541, 526; HRMS (Q-TOF) m/z: $[\mathrm{M}+\mathrm{H}]^{+}$Calcd for $\mathrm{C}_{15} \mathrm{H}_{20} \mathrm{~F}_{3} \mathrm{~N}_{4} \mathrm{O}_{4}$ : 377.1431; found: 377.1429.<smiles>COc1ccc(OC(=O)N2CCCCC2)cc1</smiles>

21

4-Methoxyphenyl piperidine-1-carboxylate (21): The title reaction was performed on $1 \mathrm{~g}$ scale with a total reaction time of 3 hours. 4-(Methoxy)phenol was activated with CDI in $10 \mathrm{~V}$ acetonitrile prior to the addition of $\mathrm{MsOH}$. Isolation via crystallization from water provided 1.61 $\mathrm{g}\left(86 \%\right.$ yield) of 21 as a white solid. ${ }^{1} \mathbf{H}$ NMR $\left(400 \mathrm{MHz} ; \mathrm{CDCl}_{3}\right) \delta 7.01(\mathrm{~d}, J=9.0 \mathrm{~Hz}, 2 \mathrm{H}), 6.86$ $(\mathrm{d}, J=8.9 \mathrm{~Hz}, 2 \mathrm{H}), 3.79(\mathrm{~s}, 3 \mathrm{H}), 3.63-3.46(\mathrm{~m}, 4 \mathrm{H}), 1.68-1.58(\mathrm{~m}, 6 \mathrm{H}) ;{ }^{13} \mathrm{C}$ NMR $(100 \mathrm{MHz}$; $\left.\mathrm{CDCl}_{3}\right) \delta 156.9,154.4,145.3,122.7,114.4,55.8,45.6,45.2,26.1,25.7,24.5$; IR (Neat) 2946, 2864, 1710, 1696, 1610, 1592, 1509, 1465, 1427, 1377, 1350, 1298, 1268, 1259, 1233, 1193, 1179 , 1139, 1104, 1056, 1032, 1005, 979, 957, 933, 909, 852, 824, 800, 751, 706, 648, 627, 564, 551, 535; HRMS (Q-TOF) m/z: [M+H] ${ }^{+}$Calcd for $\mathrm{C}_{13} \mathrm{H}_{18} \mathrm{NO}_{3}$ : 236.1281; found: 236.1281 .

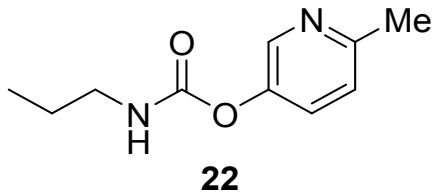

6-Methylpyridin-3-yl propylcarbamate (22): The title reaction was performed on $1 \mathrm{~g}$ scale with a total reaction time of 2 hours. 5-Hydroxy-2-methylpyridine was activated with CDI in $15 \mathrm{~V}$ acetonitrile prior to the addition of $\mathrm{MsOH}$. Purification by flash column chromatography eluting with $n$-heptane/EtOAc provided $1.19 \mathrm{~g}\left(68 \%\right.$ yield) of 22 as a clear oil. ${ }^{\mathbf{1}} \mathbf{H}$ NMR (400 MHz; $\left.\mathrm{CDCl}_{3}\right) \delta 8.29(\mathrm{~d}, J=2.7 \mathrm{~Hz}, 1 \mathrm{H}), 7.40(\mathrm{dd}, J=8.4,2.8 \mathrm{~Hz}, 1 \mathrm{H}), 7.12(\mathrm{~d}, J=8.4 \mathrm{~Hz}, 1 \mathrm{H}), 5.34$ (br s, $1 \mathrm{H}), 3.21(\mathrm{q}, J=6.6 \mathrm{~Hz}, 2 \mathrm{H}), 2.52(\mathrm{~s}, 3 \mathrm{H}), 1.62-1.52(\mathrm{~m}, 2 \mathrm{H}), 0.94(\mathrm{t}, J=7.4 \mathrm{~Hz}, 3 \mathrm{H}) ;{ }^{13} \mathrm{C}$ NMR $\left(100 \mathrm{MHz} ; \mathrm{CDCl}_{3}\right) \delta 155.0,154.2,145.9,142.3,129.8,123.5,43.1,23.7,23.1,11.3$; IR (Neat) 3324, 2964, 2875, 1720, 1603, 1535, 1483, 1383, 1206, 1156, 1124, 1030, 960, 898, 875, 829, 724, 639, 545, 535; HRMS (Q-TOF) m/z: [M+H] ${ }^{+}$Calcd for $\mathrm{C}_{10} \mathrm{H}_{15} \mathrm{~N}_{2} \mathrm{O}_{2}$ : 195.1128; found: 195.1126.

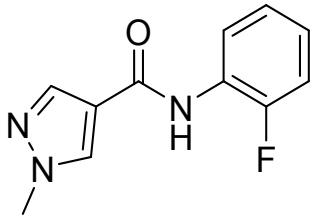

23

$\boldsymbol{N}$-(2-Fluorophenyl)-1-methyl-1H-pyrazole-4-carboxamide (23): The title reaction was performed on $1 \mathrm{~g}$ scale with a total reaction time of 2 hours. 1-Methyl-1H-pyrazole-4-carboxylic acid was activated with CDI in $10 \mathrm{~V}$ acetonitrile prior to the addition of $\mathrm{MsOH}$. Isolation via 
crystallization from water provided $1.26 \mathrm{~g}$ (74\% yield) of $\mathbf{2 3}$ as a white solid. ${ }^{1} \mathbf{H}$ NMR (400 MHz; DMSO- $\left.d_{6}\right) \delta 9.68(\mathrm{~s}, 1 \mathrm{H}), 8.32(\mathrm{~s}, 1 \mathrm{H}), 8.01(\mathrm{~s}, 1 \mathrm{H}), 7.62(\mathrm{td}, J=7.8,2.2 \mathrm{~Hz}, 1 \mathrm{H}), 7.33-7.15(\mathrm{~m}$, $3 \mathrm{H}), 3.90(\mathrm{~s}, 3 \mathrm{H}) ;{ }^{13} \mathrm{C}$ NMR $\left(100 \mathrm{MHz}\right.$; DMSO- $\left.d_{6}\right) \delta 160.5,155.4(\mathrm{~d}, J=246.1 \mathrm{~Hz}), 139.0,132.8$, $126.8(\mathrm{~d}, J=1.9 \mathrm{~Hz}), 126.4$ (d, $J=7.6 \mathrm{~Hz}), 125.6$ (d, $J=12.1 \mathrm{~Hz}), 124.2(\mathrm{~d}, J=3.5 \mathrm{~Hz}), 117.8$, 115.7 (d, $J=19.9 \mathrm{~Hz}), 38.8 ;{ }^{19} \mathbf{F}$ NMR $\left(375 \mathrm{MHz} ; \mathrm{CDCl}_{3}\right) \delta-122.0$; IR (Neat) 3309, 3109, 2947, $1661,1651,1614,1601,1556,1524,1490,1451,1404,1363,1309,1286,1257,1227,1191,1157$, 1097, 1063, 1034, 1004, 990, 930, 885, 861, 846, 776, 756, 749, 712, 695, 664, 634, 588, 553, 539, 530; HRMS (Q-TOF) m/z: [M+H] ${ }^{+}$Calcd for $\mathrm{C}_{11} \mathrm{H}_{11} \mathrm{FN}_{3} \mathrm{O}$ : 220.0881; found: 220.0880 .<smiles>Cc1cccc(C)c1NC(=O)c1ccccc1</smiles>

24

N-(2,6-Dimethylphenyl)benzamide (24): The title reaction was performed on $1 \mathrm{~g}$ scale with a total reaction time of 17 hours. Benzoic acid was activated with CDI in $10 \mathrm{~V}$ acetonitrile prior to the addition of $\mathrm{MsOH}$. Isolation via crystallization from water provided $1.68 \mathrm{~g}$ (91\% yield) of $\mathbf{2 4}$ as a white solid. Spectral data were found to be in accordance with literature data. ${ }^{1}{ }^{1} \mathbf{H}$ NMR (400 $\left.\mathrm{MHz} ; \mathrm{CDCl}_{3}\right) \delta 7.93(\mathrm{~d}, J=7.5 \mathrm{~Hz}, 2 \mathrm{H}), 7.58(\mathrm{t}, J=7.2 \mathrm{~Hz}, 1 \mathrm{H}), 7.51(\mathrm{dd}, J=7.4,7.4 \mathrm{~Hz}, 2 \mathrm{H})$, 7.36 (br s, $1 \mathrm{H}), 7.18-7.11(\mathrm{~m}, 3 \mathrm{H}), 2.30$ (s, 6H).

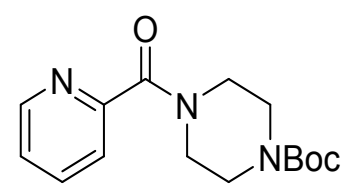

25

tert-Butyl 4-picolinoylpiperazine-1-carboxylate (25): The title reaction was performed on $1 \mathrm{~g}$ scale with a total reaction time of 2 hours. Picolinic acid was activated with CDI in $10 \mathrm{~V}$ acetonitrile prior to the addition of $\mathrm{MsOH}$. Isolation via crystallization from water provided 1.93 $\mathrm{g}$ (78\% yield) of $\mathbf{2 5}$ as a white solid. Spectral data were found to be in accordance with literature data. ${ }^{2}{ }^{1} \mathbf{H}$ NMR $\left(400 \mathrm{MHz} ; \mathrm{CDCl}_{3}\right) \delta 8.59(\mathrm{~d}, J=4.4 \mathrm{~Hz}, 1 \mathrm{H}), 7.81(\mathrm{td}, J=7.7,1.7 \mathrm{~Hz}, 1 \mathrm{H}), 7.67$ $(\mathrm{d}, J=7.8 \mathrm{~Hz}, 1 \mathrm{H}), 7.35(\mathrm{ddd}, J=7.6,4.9,1.1 \mathrm{~Hz}, 1 \mathrm{H}), 3.82-3.69(\mathrm{~m}, 2 \mathrm{H}), 3.57(\mathrm{dt}, J=17.1$, $4.6 \mathrm{~Hz}, 4 \mathrm{H}), 3.51-3.43(\mathrm{~m}, 2 \mathrm{H}), 1.47$ (s, 9H).

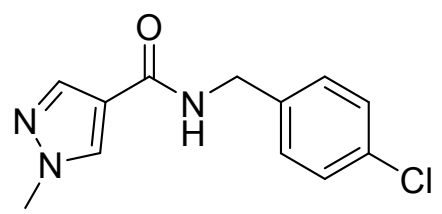

26

$\boldsymbol{N}$-(4-Chlorobenzyl)-1-methyl-1H-pyrazole-4-carboxamide (26): The title reaction was performed on $1 \mathrm{~g}$ scale with a total reaction time of 3 hours. 1-Methyl-1H-pyrazole-4-carboxylic acid was activated with CDI in $10 \mathrm{~V}$ acetonitrile prior to the addition of $\mathrm{MsOH}$. Isolation via crystallization from water provided $1.49 \mathrm{~g}$ (78\% yield) of $\mathbf{2 6}$ as a white solid. ${ }^{\mathbf{1}} \mathbf{H}$ NMR (400 MHz; $\left.\mathrm{CDCl}_{3}\right) \delta 7.83(\mathrm{~s}, 1 \mathrm{H}), 7.71(\mathrm{~s}, 1 \mathrm{H}), 7.35-7.21(\mathrm{~m}, 4 \mathrm{H}), 6.06(\mathrm{br} \mathrm{s}, 1 \mathrm{H}), 4.55(\mathrm{~d}, J=5.9 \mathrm{~Hz}, 2 \mathrm{H})$, 
$3.92(\mathrm{~s}, 3 \mathrm{H}) ;{ }^{13} \mathrm{C}$ NMR $\left(100 \mathrm{MHz} ; \mathrm{CDCl}_{3}\right) \delta 162.5,137.8,137.0,133.5,132.0,129.4,129.0$, 118.6, 42.9, 39.5; IR (Neat) 3265, 2928, 1624, 1565, 1534, 1491, 1425, 1405, 1343, 1301, 1269, $1235,1109,1089,1045,1016,987,948,872,844,825,803,786,766,725,700,657,643,603$, 578, 570, 554, 542, 530; HRMS (Q-TOF) $\mathbf{~ m / z : ~}[\mathrm{M}+\mathrm{H}]^{+}$Calcd for $\mathrm{C}_{12} \mathrm{H}_{13} \mathrm{ClN}_{3} \mathrm{O}: 250.0742$; found: 250.0740 .

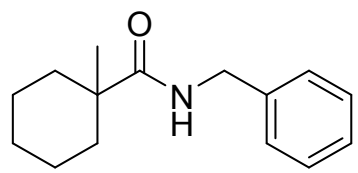

27

$\mathrm{N}$-Benzyl-1-methylcyclohexane-1-carboxamide (27): The title reaction was performed on $1 \mathrm{~g}$ scale with a total reaction time of 2 hours. 1-Methyl-1-cyclohexanecarboxylic acid was activated with $\mathrm{CDI}$ in $10 \mathrm{~V}$ acetonitrile prior to the addition of $\mathrm{MsOH}$. Isolation via crystallization from water provided $1.36 \mathrm{~g}$ ( $85 \%$ yield) of $\mathbf{2 7}$ as a white solid. ${ }^{1} \mathbf{H}$ NMR $\left(400 \mathrm{MHz}\right.$; DMSO- $\left.d_{6}\right) \delta 8.06$ (t, $J=6.1 \mathrm{~Hz}, 1 \mathrm{H}), 7.31(\mathrm{td}, J=7.1,1.6 \mathrm{~Hz}, 2 \mathrm{H}), 7.26-7.19(\mathrm{~m}, 3 \mathrm{H}), 4.29(\mathrm{~d}, J=6.0 \mathrm{~Hz}, 2 \mathrm{H})$, $2.04-1.93$ (m, 2H), $1.54-1.41$ (m, 3H), $1.42-1.14$ (m, 5H), 1.08 (s, 3H); ${ }^{13} \mathbf{C}$ NMR (100 MHz; DMSO- $\left.d_{6}\right) \delta 177.0,140.9,128.6,127.3,126.9,42.54,42.45,35.6,27.0,25.9,23.2$; IR (Neat) $3322,2923,2858,1628,1530,1495,1470,1444,1427,1322,1268,1250,1239,1176,1143,1106$, 1081, 1030, 997, 962, 930, 849, 821, 747, 699, 663, 608, 564, 543, 535; HRMS (Q-TOF) m/z: $[\mathrm{M}+\mathrm{H}]^{+}$Calcd for $\mathrm{C}_{15} \mathrm{H}_{22} \mathrm{NO}$ : 232.1696; found: 232.1696.

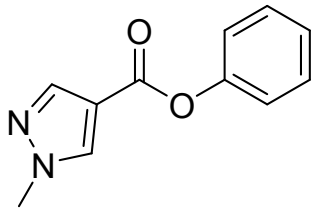

28

Phenyl 1-methyl-1H-pyrazole-4-carboxylate (28): The title reaction was performed on $1 \mathrm{~g}$ scale with a total reaction time of 93 hours. 1-Methyl-1H-pyrazole-4-carboxylic acid was activated with $\mathrm{CDI}$ in $10 \mathrm{~V}$ acetonitrile prior to the addition of $\mathrm{MsOH}$. Purification by flash column chromatography eluting with $n$-heptane/EtOAc (60:40) provided $0.98 \mathrm{~g}$ (63\% yield) of $\mathbf{2 8}$ as a white solid. Spectral data were found to be in accordance with literature data. ${ }^{3}{ }^{1} \mathbf{H}$ NMR (400 $\left.\mathrm{MHz} ; \mathrm{CDCl}_{3}\right) \delta 8.05(\mathrm{~s}, 1 \mathrm{H}), 8.02(\mathrm{~s}, 1 \mathrm{H}), 7.41(\mathrm{dd}, J=7.9,7.9 \mathrm{~Hz}, 2 \mathrm{H}), 7.33-7.21(\mathrm{~m}, 1 \mathrm{H})$, $7.17(\mathrm{~d}, J=8.3 \mathrm{~Hz}, 2 \mathrm{H}), 3.98(\mathrm{~s}, 3 \mathrm{H})$.

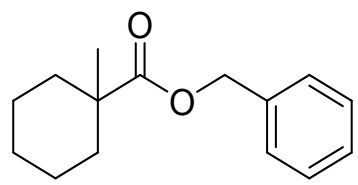

29

Benzyl 1-methylcyclohexane-1-carboxylate (29): The title reaction was performed on $1 \mathrm{~g}$ scale with a total reaction time of 41 hours. 1-Methyl-1-cyclohexanecarboxylic acid was activated with $\mathrm{CDI}$ in $10 \mathrm{~V}$ acetonitrile prior to the addition of $\mathrm{MsOH}$. Purification by flash column chromatography eluting with $n$-heptane/EtOAc (90:10) provided $1.31 \mathrm{~g}$ (80\% yield) of 29 as a 
clear oil. Spectral data were found to be in accordance with literature data. ${ }^{4} \mathbf{H}$ NMR (400 MHz; $\left.\mathrm{CDCl}_{3}\right) \delta$ 7.39-7.27 (m, 5H), $5.13(\mathrm{~s}, 2 \mathrm{H}), 2.16-1.99(\mathrm{~m}, 2 \mathrm{H}), 1.57-1.47(\mathrm{~m}, 3 \mathrm{H}), 1.42-1.20$ $(\mathrm{m}, 5 \mathrm{H}), 1.18(\mathrm{~s}, 3 \mathrm{H})$.<smiles>O=C(OC1CCCCC1)c1ccccc1</smiles>

30

Cyclohexyl benzoate (30): The title reaction was performed on $1 \mathrm{~g}$ scale with a total reaction time of 21 hours. Benzoic acid was activated with CDI in $10 \mathrm{~V}$ acetonitrile prior to the addition of $\mathrm{MsOH}$. Purification by flash column chromatography eluting with $n$-heptane/EtOAc (95:5) provided $0.99 \mathrm{~g}$ ( $60 \%$ yield) of $\mathbf{3 0}$ as a clear oil. Spectral data were found to be in accordance with literature data. ${ }^{5}{ }^{1} \mathrm{H}$ NMR $\left(400 \mathrm{MHz} ; \mathrm{CDCl}_{3}\right) \delta 8.05(\mathrm{~d}, J=8.0 \mathrm{~Hz}, 2 \mathrm{H}), 7.55(\mathrm{t}, J=6.9 \mathrm{~Hz}, 1 \mathrm{H})$, $7.43(\mathrm{dd}, J=7.7,7.7 \mathrm{~Hz}, 2 \mathrm{H}), 5.04(\mathrm{tt}, J=8.4,3.6 \mathrm{~Hz}, 1 \mathrm{H}), 2.05-1.89(\mathrm{~m}, 2 \mathrm{H}), 1.89-1.74(\mathrm{~m}$, $2 \mathrm{H}), 1.66-1.54(\mathrm{~m}, 3 \mathrm{H}), 1.53-1.29(\mathrm{~m}, 3 \mathrm{H})$.<smiles>COc1ccc(OC(=O)c2ccccc2)cc1</smiles>

31

4-Methoxyphenyl benzoate (31): The title reaction was performed on $1 \mathrm{~g}$ scale with a total reaction time of 21 hours. Benzoic acid was activated with CDI in $10 \mathrm{~V}$ acetonitrile prior to the addition of $\mathrm{MsOH}$. Isolation via crystallization from water provided $1.66 \mathrm{~g} \mathrm{(90 \%} \mathrm{yield)} \mathrm{of} \mathbf{3 1}$ as a white solid. Spectral data were found to be in accordance with literature data. ${ }^{6}{ }^{1} \mathbf{H}$ NMR (400 $\left.\mathrm{MHz} ; \mathrm{CDCl}_{3}\right) \delta 8.20(\mathrm{~d}, J=7.9 \mathrm{~Hz}, 2 \mathrm{H}), 7.63(\mathrm{t}, J=7.4 \mathrm{~Hz}, 1 \mathrm{H}), 7.52(\mathrm{dd}, J=7.6,7.6 \mathrm{~Hz}, 2 \mathrm{H})$, $7.13(\mathrm{~d}, J=9.0 \mathrm{~Hz}, 2 \mathrm{H}), 6.94(\mathrm{~d}, J=8.9 \mathrm{~Hz}, 2 \mathrm{H}), 3.83(\mathrm{~s}, 3 \mathrm{H})$.<smiles>CCCCOC(=O)c1ccccn1</smiles>

32

Butyl picolinate (32): The title reaction was performed on $1 \mathrm{~g}$ scale with a total reaction time of 17 hours. Picolinic acid was activated with $\mathrm{CDI}$ in $10 \mathrm{~V}$ acetonitrile prior to the addition of $\mathrm{MsOH}$. Purification by flash column chromatography eluting with $n$-heptane/EtOAc (80:20) provided 1.14 $\mathrm{g}$ (79\% yield) of $\mathbf{3 2}$ as a yellow oil. Spectral data were found to be in accordance with literature data. ${ }^{7} \mathbf{H}$ NMR $\left(400 \mathrm{MHz} ; \mathrm{CDCl}_{3}\right) \delta 8.76(\mathrm{~d}, J=4.5 \mathrm{~Hz}, 1 \mathrm{H}), 8.12(\mathrm{~d}, J=7.8 \mathrm{~Hz}, 1 \mathrm{H}), 7.83(\mathrm{dd}$, $J=7.7,7.7 \mathrm{~Hz}, 1 \mathrm{H}), 7.46(\mathrm{dd}, J=7.4,4.9 \mathrm{~Hz}, 1 \mathrm{H}), 4.42(\mathrm{t}, J=6.9 \mathrm{~Hz}, 2 \mathrm{H}), 1.85-1.76(\mathrm{~m}, 2 \mathrm{H})$, $1.51-1.42(\mathrm{~m}, 2 \mathrm{H}), 0.97(\mathrm{t}, J=7.4 \mathrm{~Hz}, 3 \mathrm{H})$. 


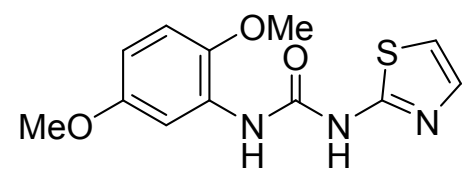

33

1-(2,5-Dimethoxyphenyl)-3-(thiazol-2-yl)urea (33): The title reaction was performed on $1 \mathrm{~g}$ scale with a total reaction time of 1 hour. 2-Aminothiazole was activated with CDI in $15 \mathrm{~V}$ acetonitrile prior to the addition of $\mathrm{MsOH}$. Isolation via crystallization from water provided 2.21 $\mathrm{g}(82 \%$ yield $)$ of $\mathbf{3 3}$ as a grey solid. Spectral data were found to be in accordance with literature data. ${ }^{8}{ }^{1} \mathbf{H}$ NMR (400 MHz; DMSO-d $\left.)\right) \delta 11.06$ (br s, $\left.1 \mathrm{H}\right), 8.77$ (br s, $\left.1 \mathrm{H}\right), 7.81(\mathrm{~d}, J=2.6 \mathrm{~Hz}, 1 \mathrm{H})$, $7.38(\mathrm{~d}, J=3.4 \mathrm{~Hz}, 1 \mathrm{H}), 7.12(\mathrm{~d}, J=3.3 \mathrm{~Hz}, 1 \mathrm{H}), 6.95$ (d, $J=8.9 \mathrm{~Hz}, 1 \mathrm{H}), 6.55$ (dd, $J=8.8,2.7$ $\mathrm{Hz}, 1 \mathrm{H}), 3.82(\mathrm{~s}, 3 \mathrm{H}), 3.70(\mathrm{~s}, 3 \mathrm{H})$.<smiles>CN(C(=O)NCc1ccccc1)C1CCCCC1</smiles>

34

3-Benzyl-1-cyclohexyl-1-methylurea (34): The title reaction was performed on $1 \mathrm{~g}$ scale with a total reaction time of 1 hour. Benzylamine was activated with CDI in $15 \mathrm{~V}$ acetonitrile prior to the addition of $\mathrm{MsOH}$. Purification by flash column chromatography eluting with $n$-heptane/EtOAc provided $1.23 \mathrm{~g}$ (54\% yield) of $\mathbf{3 4}$ as a white solid. ${ }^{1} \mathbf{H}$ NMR $\left(400 \mathrm{MHz}\right.$; DMSO- $\left.d_{6}\right) \delta 7.32-7.16$ $(\mathrm{m}, 5 \mathrm{H}), 6.75(\mathrm{t}, J=5.8 \mathrm{~Hz}, 1 \mathrm{H}), 4.24(\mathrm{~d}, J=5.9 \mathrm{~Hz}, 2 \mathrm{H}), 3.90(\mathrm{tt}, J=11.5,3.7 \mathrm{~Hz}, 1 \mathrm{H}), 2.68(\mathrm{~s}$, $3 \mathrm{H}), 1.77-1.68(\mathrm{~m}, 2 \mathrm{H}), 1.64-1.48(\mathrm{~m}, 3 \mathrm{H}), 1.45-1.19(\mathrm{~m}, 4 \mathrm{H}), 1.13-0.98(\mathrm{~m}, 1 \mathrm{H}) ;{ }^{13} \mathbf{C} \mathbf{N M R}$ $\left(100 \mathrm{MHz} ; \mathrm{CDCl}_{3}\right)$ 157.6, 141.4, 128.0, 126.9, 126.2, 52.8, 43.5, 29.9, 27.8, 25.4, 25.1; IR (Neat) $3348,2924,2852,1622,1529,1494,1480,1450,1412,1391,1347,1313,1261,1232,1201,1179$, 1091, 1069, 1055, 1037, 1028, 1004, 962, 895, 827, 787, 769, 724, 696, 622, 604, 570, 542, 535, 530; HRMS (Q-TOF) m/z: [M+H] ${ }^{+}$Calcd for $\mathrm{C}_{15} \mathrm{H}_{23} \mathrm{~N}_{2} \mathrm{O}$ : 247.1805; found: 247.1804.

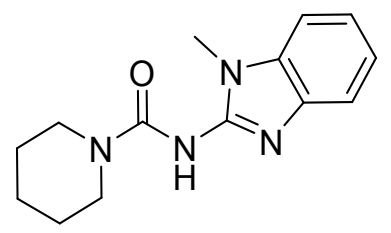

35

$N$-(1-Methyl-1H-benzo[ $d]$ imidazol-2-yl)piperidine-1-carboxamide (35): The title reaction was performed on $1 \mathrm{~g}$ scale with a total reaction time of 19 hours. 2-Amino-1-methylbenzimidazole was activated with $\mathrm{CDI}$ in $15 \mathrm{~V}$ acetonitrile prior to the addition of $\mathrm{MsOH}$. Isolation via crystallization from water provided $1.42 \mathrm{~g}$ (82\% yield) of $\mathbf{3 5}$ as an orange solid. ${ }^{\mathbf{1}} \mathbf{H}$ NMR (400 MHz; DMSO- $\left.d_{6}\right) \delta 11.93$ (br s, $\left.1 \mathrm{H}\right), 7.32(\mathrm{~d}, J=7.2 \mathrm{~Hz}, 1 \mathrm{H}), 7.25(\mathrm{~d}, J=7.7 \mathrm{~Hz}, 1 \mathrm{H}), 7.19-6.99$ $(\mathrm{m}, 2 \mathrm{H}), 3.73(\mathrm{~s}, 4 \mathrm{H}), 3.47(\mathrm{~s}, 3 \mathrm{H}), 1.67-1.52(\mathrm{~m}, 2 \mathrm{H}), 1.52-1.39(\mathrm{~m}, 4 \mathrm{H}) ;{ }^{13} \mathbf{C}$ NMR $(100 \mathrm{MHz}$; $\left.\mathrm{CDCl}_{3}\right) \delta 163.6,154.3,130.9,129.0,122.03,121.97,110.0,108.1,27.9,26.2,25.1$ (two carbons observed by HMBC); IR (Neat) 3255, 2918, 2849, 1602, 1571, 1490, 1465, 1417, 1396, 1381, $1350,1328,1269,1240,1212,1180,1147,1121,1092,1076,1028,1009,989,956,923,903$, 889, 852, 774, 731, 701, 653, 569, 549, 537; HRMS (Q-TOF) m/z: $[\mathrm{M}+\mathrm{H}]^{+}$Calcd for $\mathrm{C}_{14} \mathrm{H}_{19} \mathrm{~N}_{4} \mathrm{O}$ : 
259.1553; found: 259.1555 .<smiles>CC(C)(C)OC(=O)Nc1ccc(NC(=O)Nc2ccc3[nH]ccc3c2)cc1</smiles>

tert-Butyl (4-(3-(1H-indol-5-yl)ureido)phenyl)carbamate (36): The title reaction was performed on $1 \mathrm{~g}$ scale with a total reaction time of 4 hours. $N$-Boc- $p$-phenylenediamine was activated with $\mathrm{CDI}$ in $15 \mathrm{~V}$ acetonitrile prior to the addition of $\mathrm{MsOH}$. Isolation via crystallization from water provided $1.48 \mathrm{~g}$ ( $87 \%$ yield) of $\mathbf{3 6}$ as a grey solid. Spectral data were found to be in accordance with literature data. ${ }^{8}{ }^{1} \mathbf{H}$ NMR $\left(400 \mathrm{MHz}\right.$; DMSO- $\left.d_{6}\right) \delta 10.92$ (br s, $\left.1 \mathrm{H}\right), 9.15$ (br s, $\left.1 \mathrm{H}\right), 8.39$ (s, $1 \mathrm{H}), 8.30(\mathrm{~s}, 1 \mathrm{H}), 7.66(\mathrm{~d}, J=1.7 \mathrm{~Hz}, 1 \mathrm{H}), 7.38-7.24(\mathrm{~m}, 6 \mathrm{H}), 7.05(\mathrm{dd}, J=8.7,1.9 \mathrm{~Hz}, 1 \mathrm{H})$, 6.35-6.32 (m, 1H), $1.47(\mathrm{~s}, 9 \mathrm{H})$.<smiles>COc1cc(CNC(=O)NCc2ccccc2)cc(OC)c1</smiles>

37

1-Benzyl-3-(3,5-dimethoxybenzyl)urea (37): The title reaction was performed on $1 \mathrm{~g}$ scale with a total reaction time of 3 hours. Benzylamine was activated with CDI in $15 \mathrm{~V}$ acetonitrile prior to the addition of $\mathrm{MsOH}$. Isolation via crystallization from water provided $2.52 \mathrm{~g}$ ( $90 \%$ yield) of 37 as a white solid. ${ }^{1} \mathbf{H}$ NMR $\left(400 \mathrm{MHz} ; \mathrm{CDCl}_{3}\right) \delta 7.38-7.21(\mathrm{~m}, 5 \mathrm{H}), 6.43(\mathrm{~d}, J=1.7 \mathrm{~Hz}, 2 \mathrm{H})$, 6.37-6.34 (m, 1H), 4.69-4.60 (m, 2H), 4.39 (d, $J=5.7 \mathrm{~Hz}, 2 \mathrm{H}), 4.32$ (d, $J=5.8 \mathrm{~Hz}, 2 \mathrm{H}), 3.76(\mathrm{~s}$, $6 \mathrm{H}) ;{ }^{13} \mathbf{C}$ NMR $\left(100 \mathrm{MHz} ; \mathrm{CDCl}_{3}\right) \delta 161.2,158.0,141.6,139.2,128.8,127.6,127.5,105.4,99.5$, 55.5, 45.0, 44.8; IR (Neat) 3321, 2876, 2836, 1612, 1598, 1569, 1469, 1453, 1428, 1371, 1353, 1336, 1296, 1242, 1203, 1201, 1153, 1070, 1058, 1048, 986, 923, 838, 818, 796, 774, 756, 698, 637, 587, 572, 563, 534, 528; HRMS (Q-TOF) m/z: [M+H] ${ }^{+}$Calcd for $\mathrm{C}_{17} \mathrm{H}_{21} \mathrm{~N}_{2} \mathrm{O}_{3}: 301.1547$; found: 301.1544 .

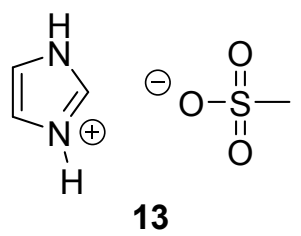

1H-Imidazol-3-ium methanesulfonate (13): The solids from the slurry generated following the addition of $\mathrm{MsOH}$ (1.0 equiv) were isolated via filtration to provide $\mathbf{1 3}$ as a white solid. ${ }^{1} \mathbf{H}$ NMR $\left(400 \mathrm{MHz}\right.$; DMSO- $\left.d_{6}\right) \delta 14.33$ (br s, 2H), 9.09 (s, 1H), 7.69 (s, 2H), 2.37 (s, 3H); ${ }^{13}$ C NMR (100 MHz; DMSO- $d_{6}$ ) $\delta 134.4,119.3,39.8$; IR (Neat) 3144, 2974, 2856, 1587, 1457, 1434, 1337, 1220 , 1156, 1115, 1092, 1031, 917, 830, 769, 626, 550, 528. 


\section{NMR Spectra}<smiles>O=C(Oc1ccc(C(F)(F)F)cc1)N1CCCCC1</smiles>

15

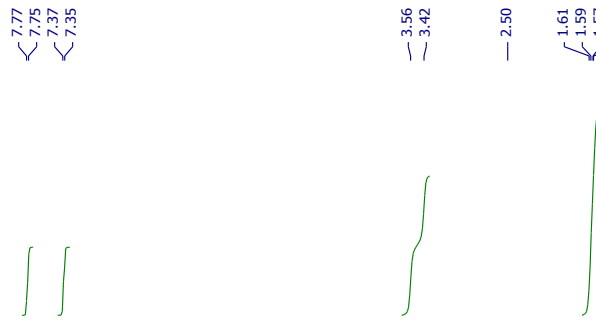

0.40

0.30
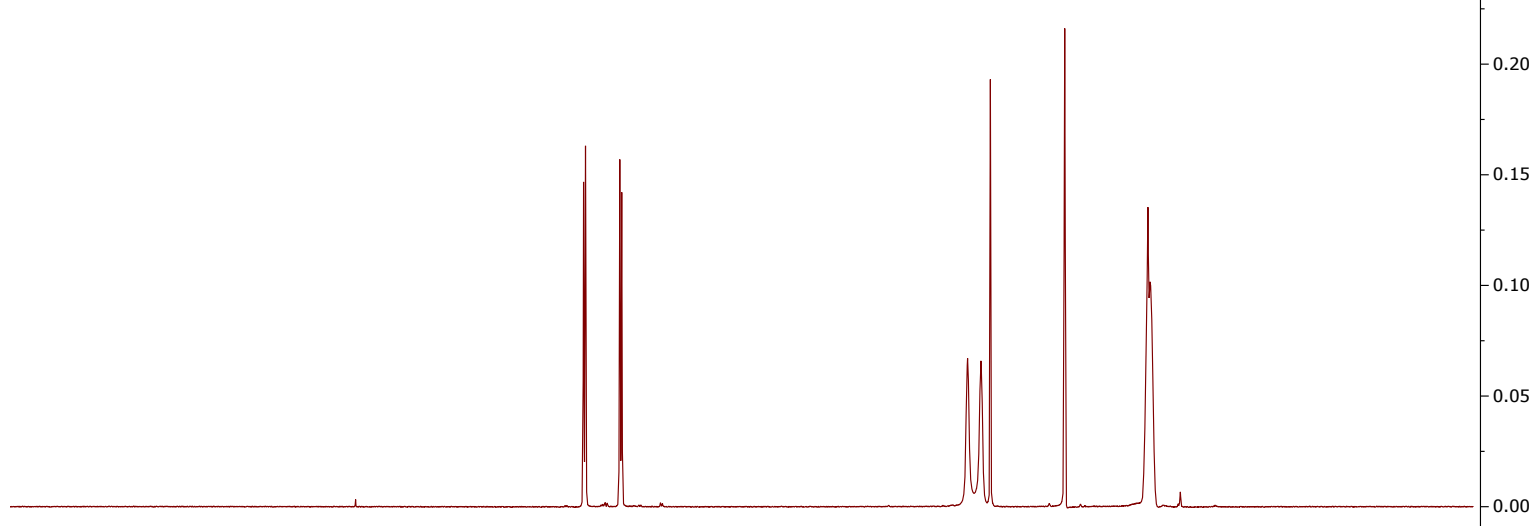

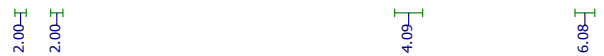

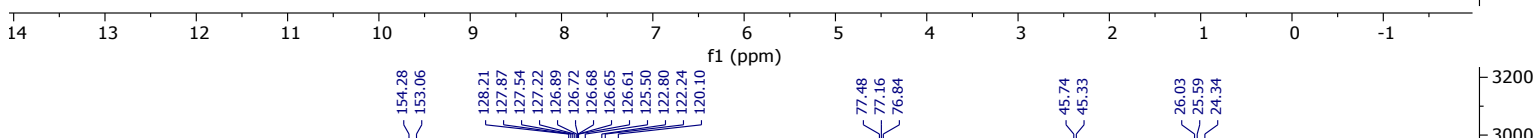

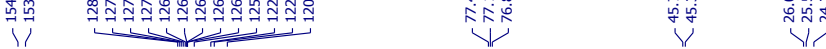

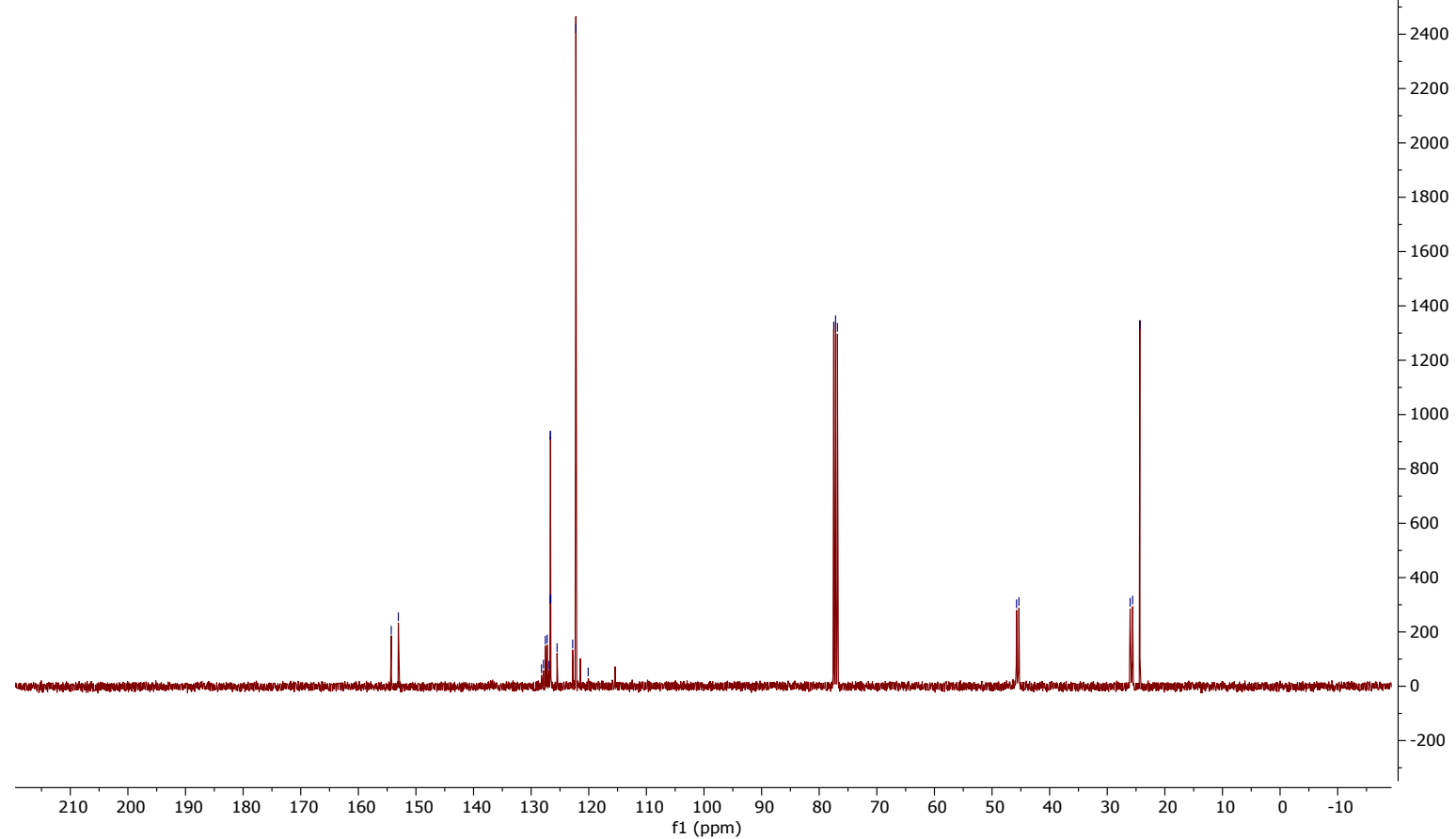




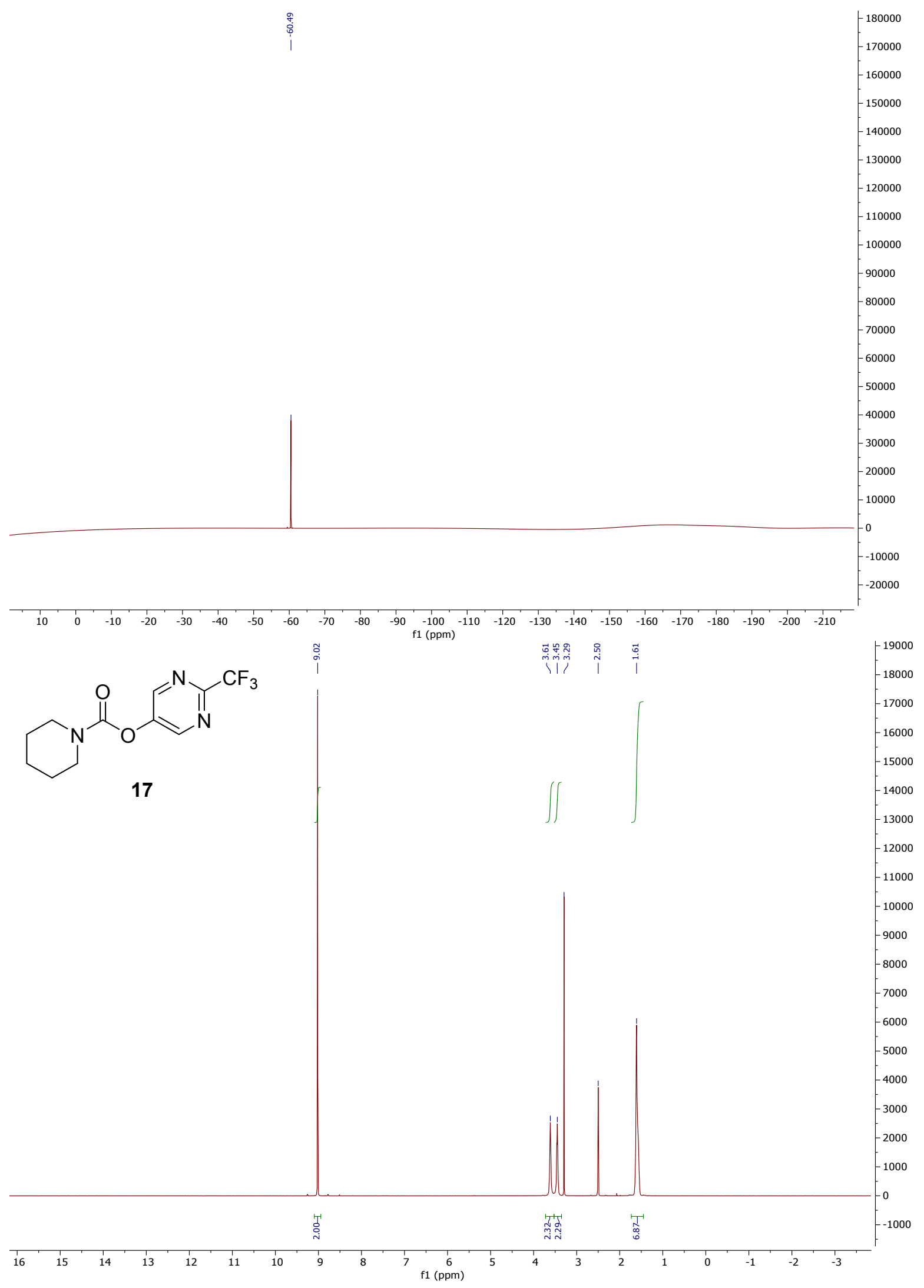




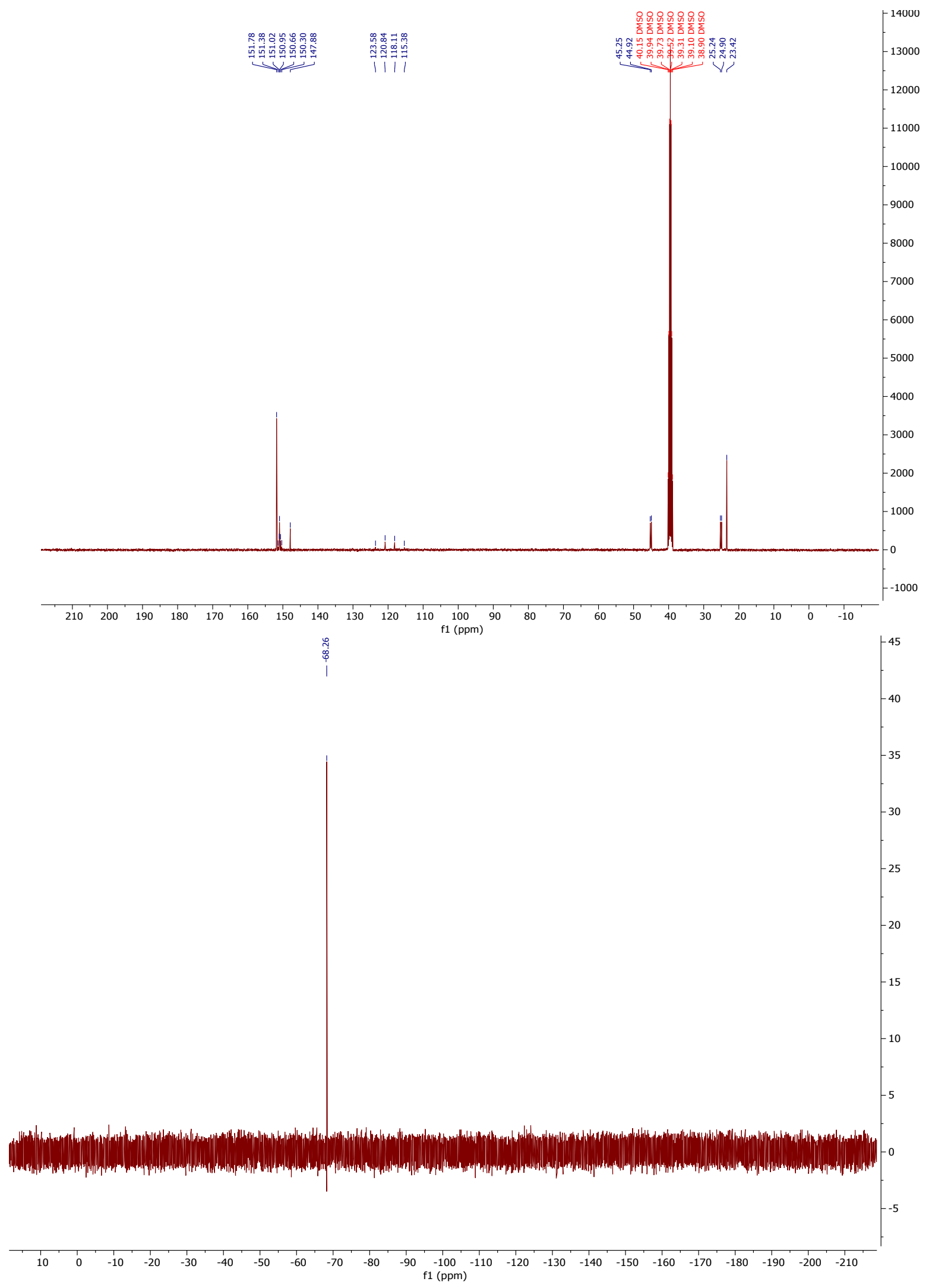




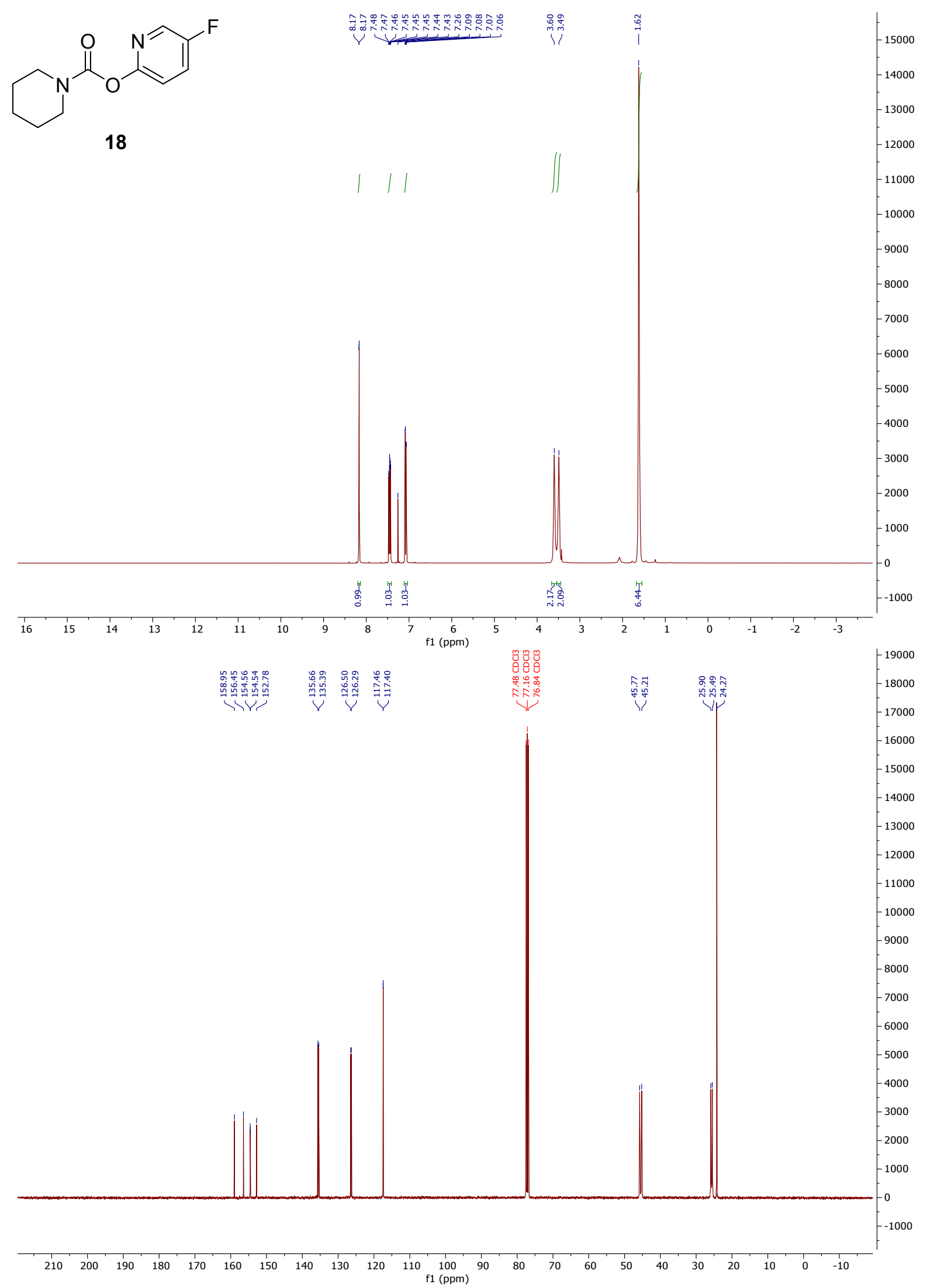




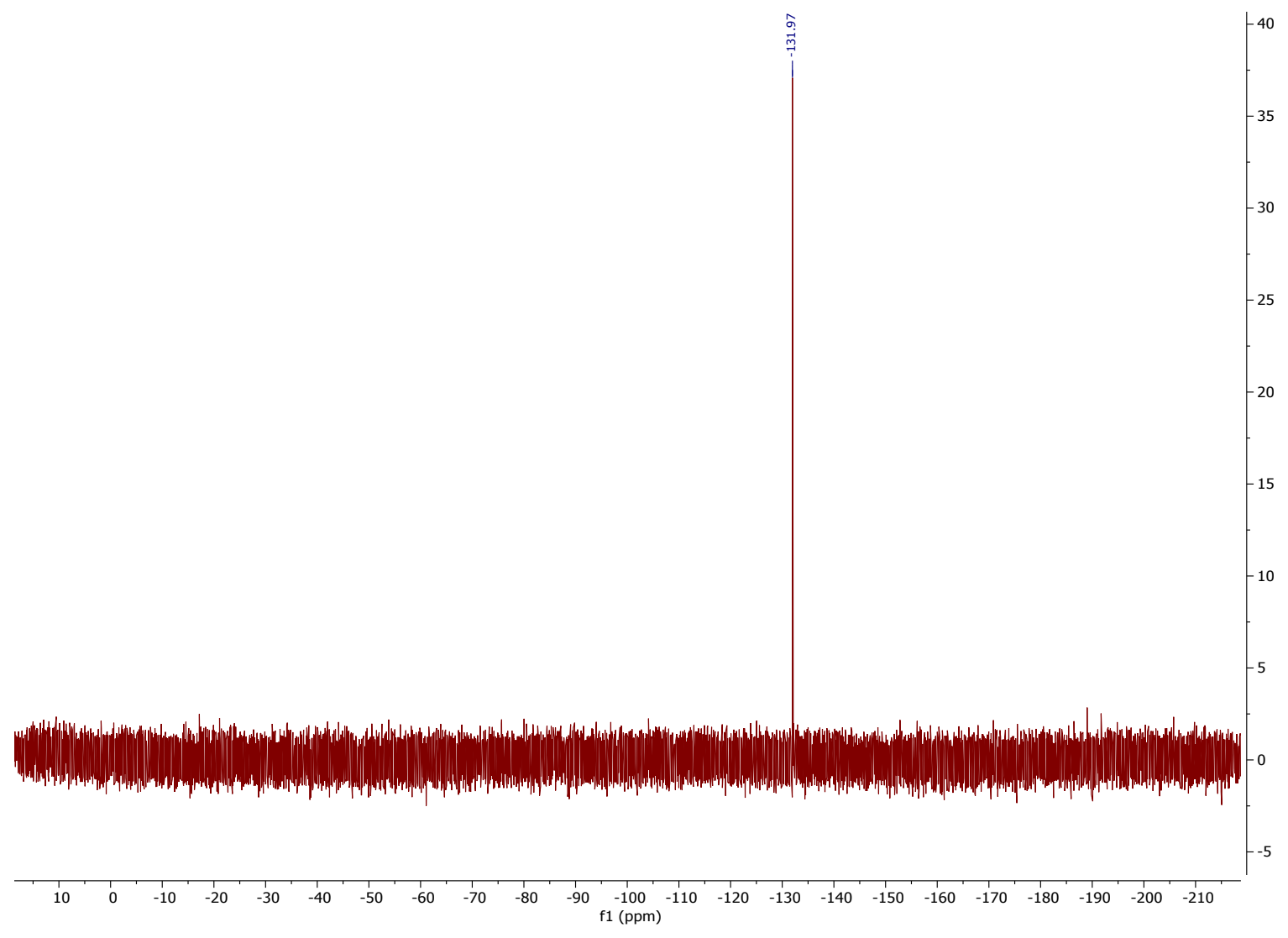<smiles>O=C(Oc1c(F)c(F)c(F)c(F)c1F)N1CCCCC1</smiles>

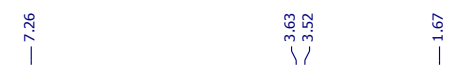

19

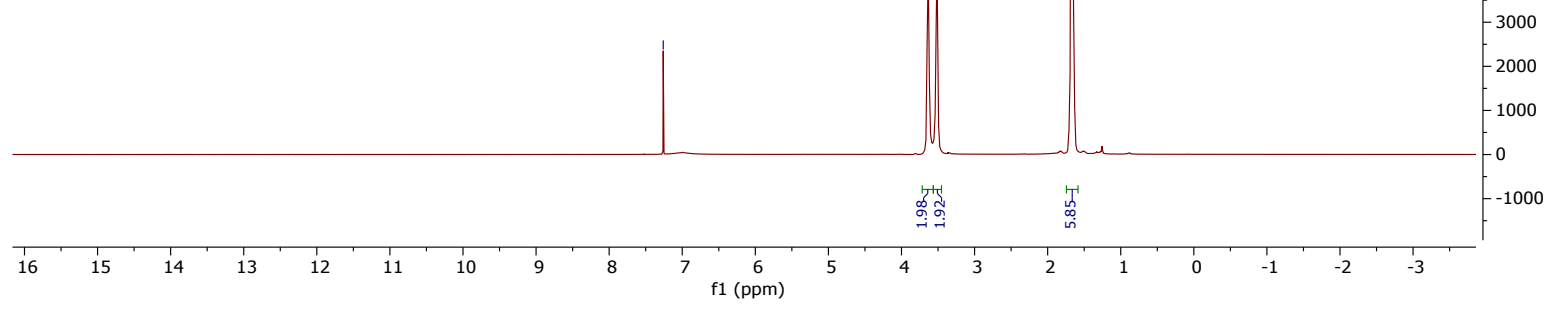




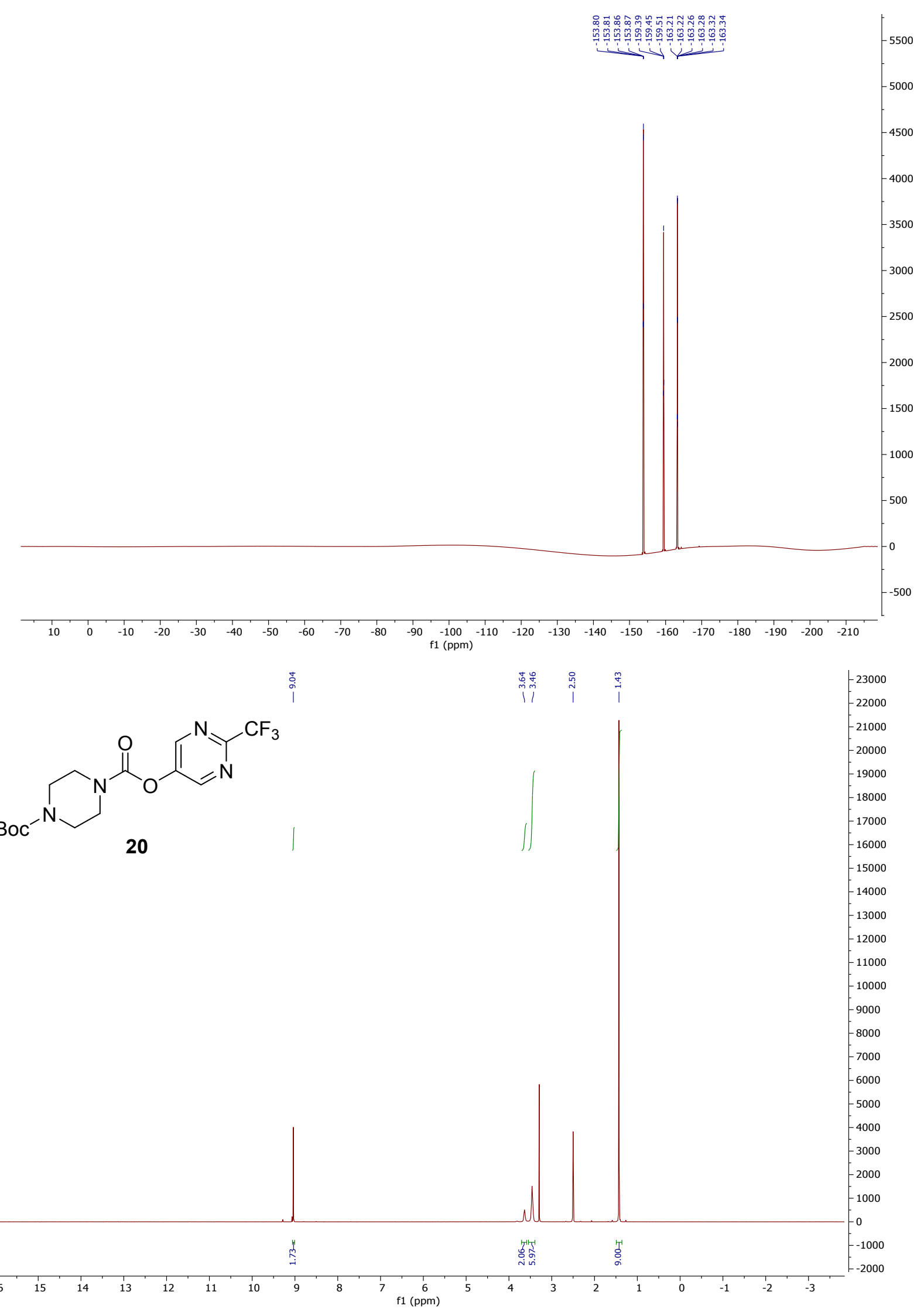




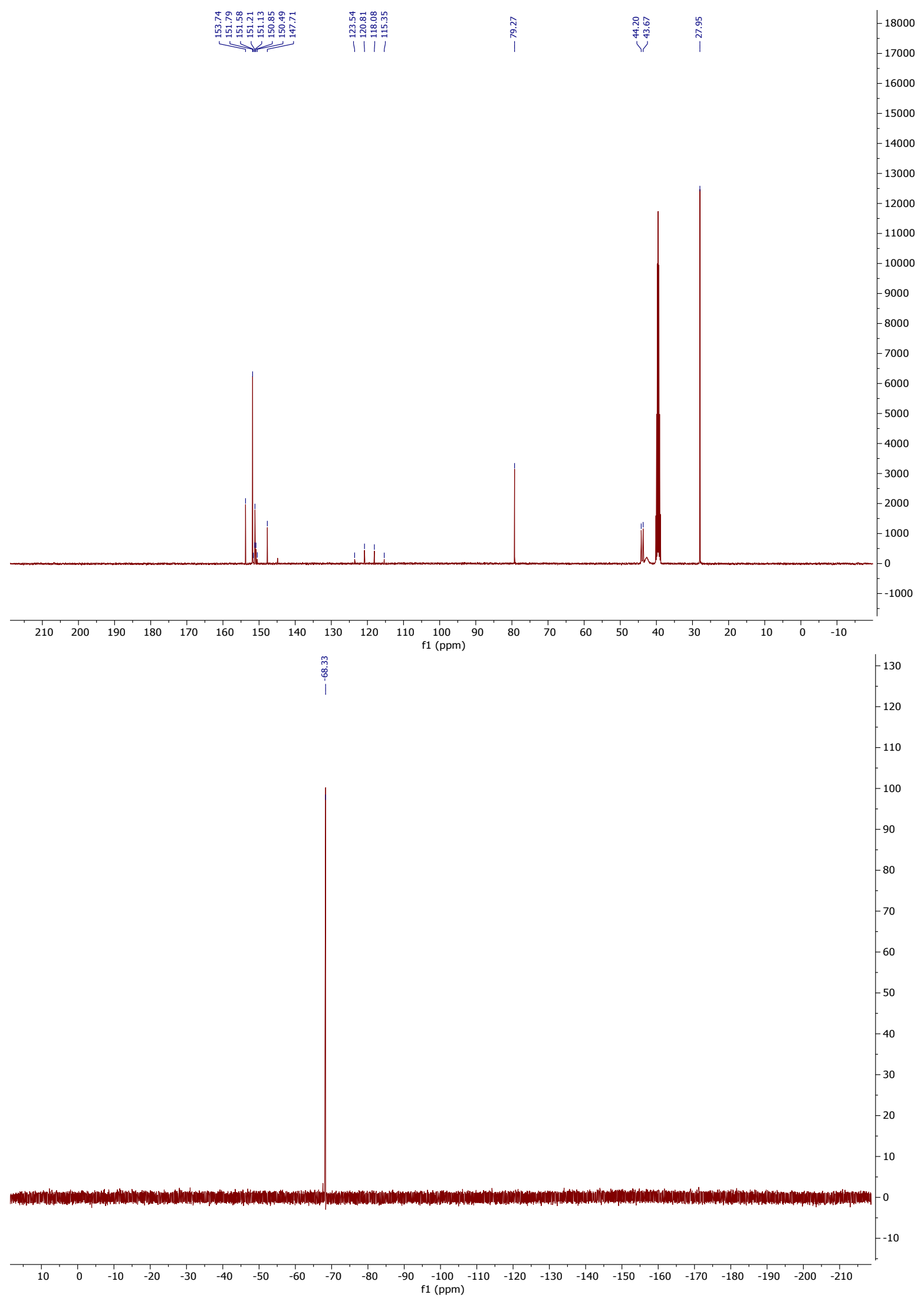




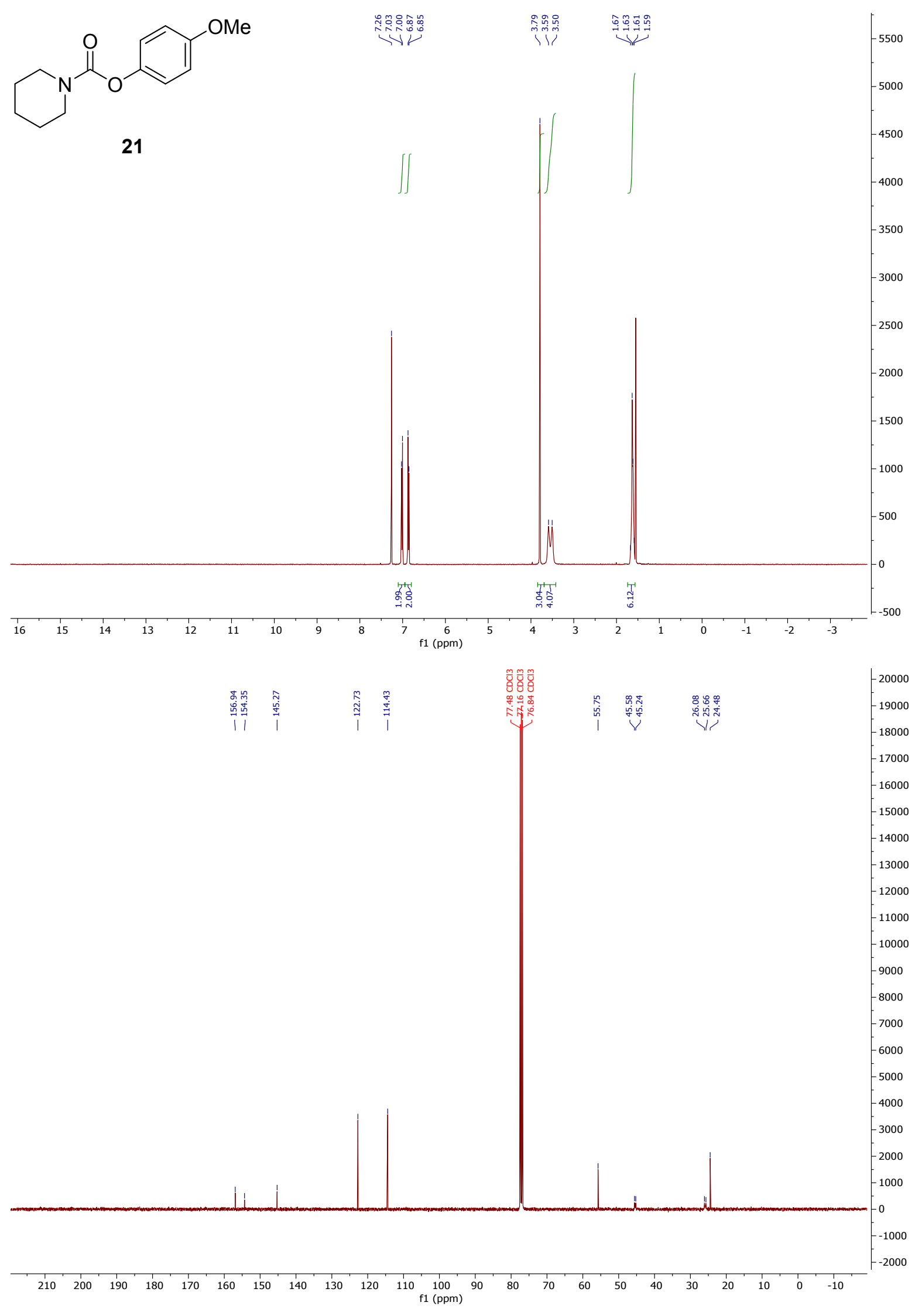



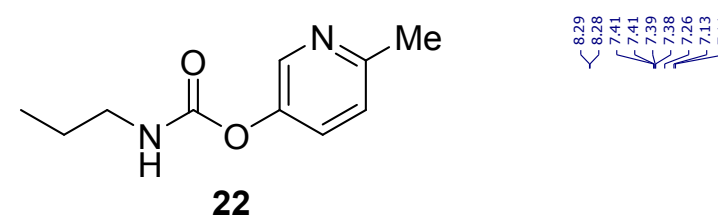

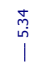
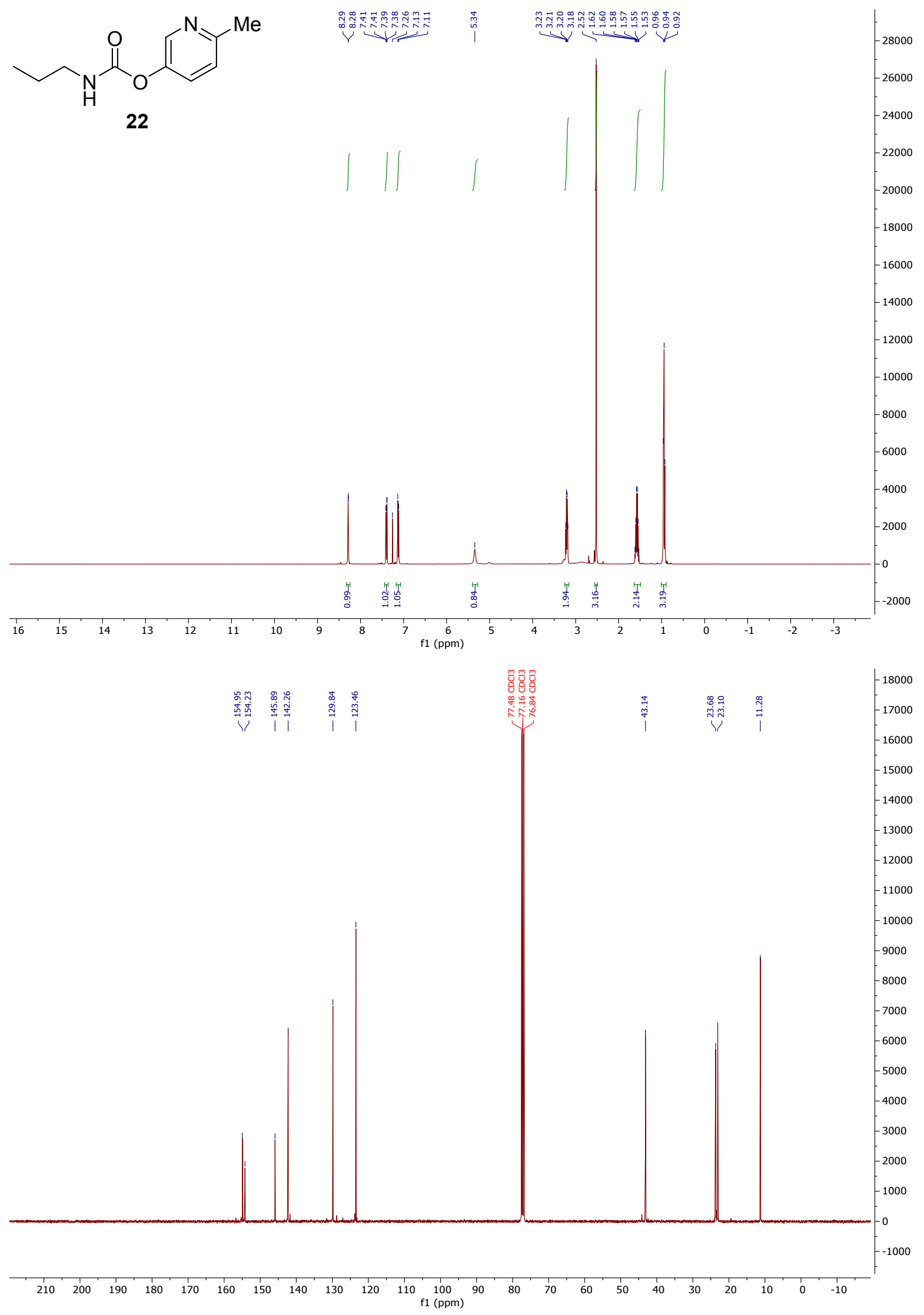


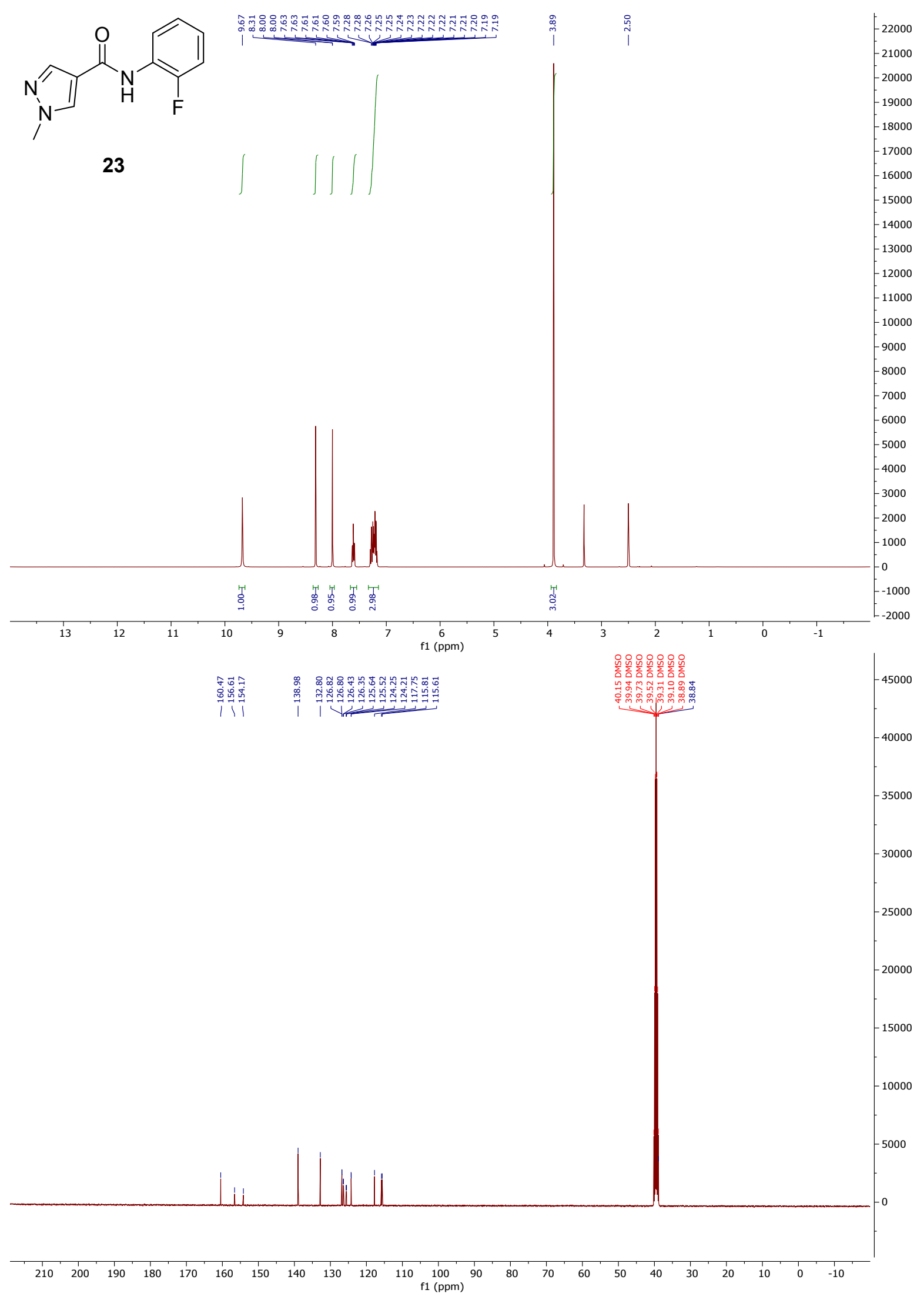




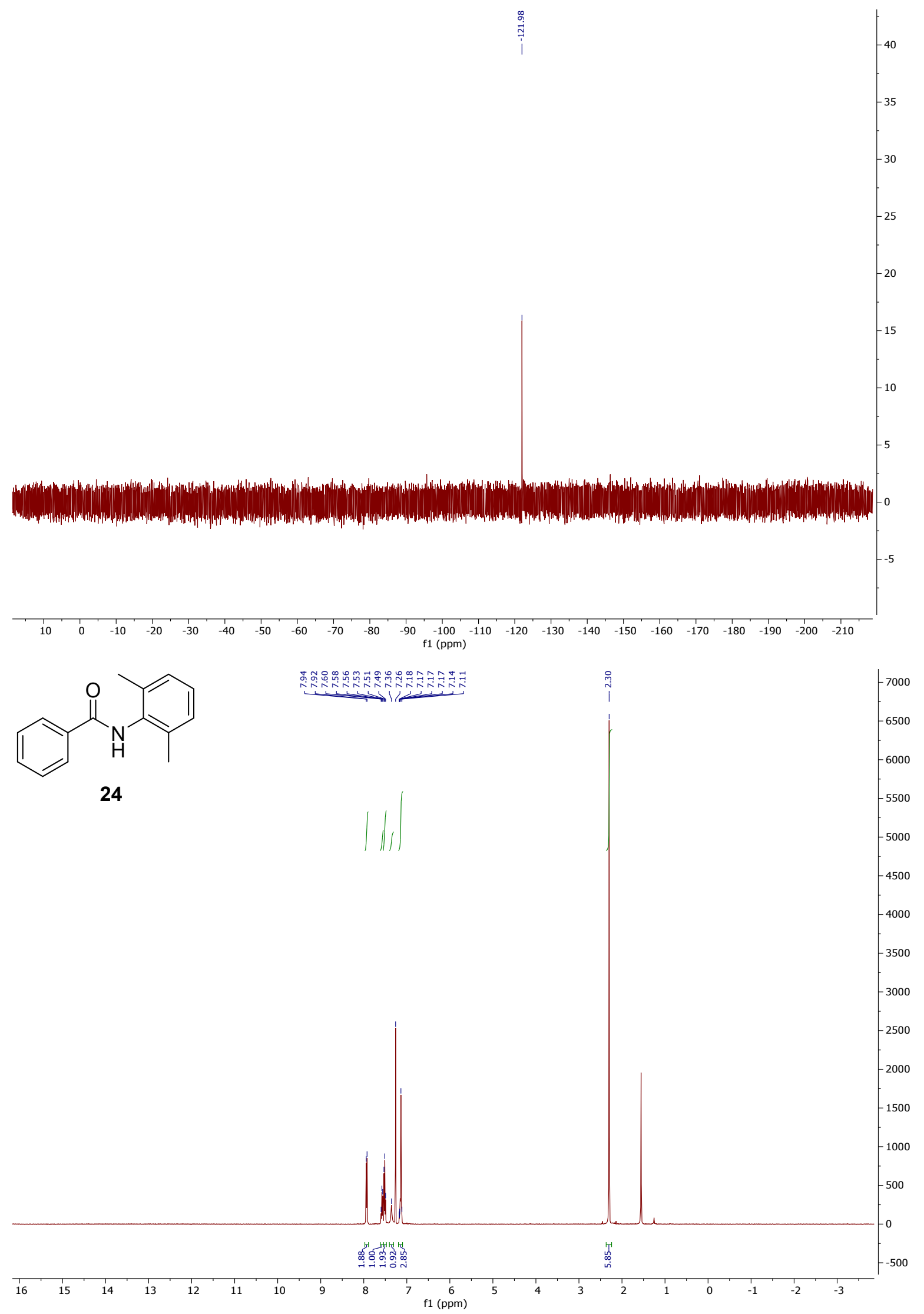




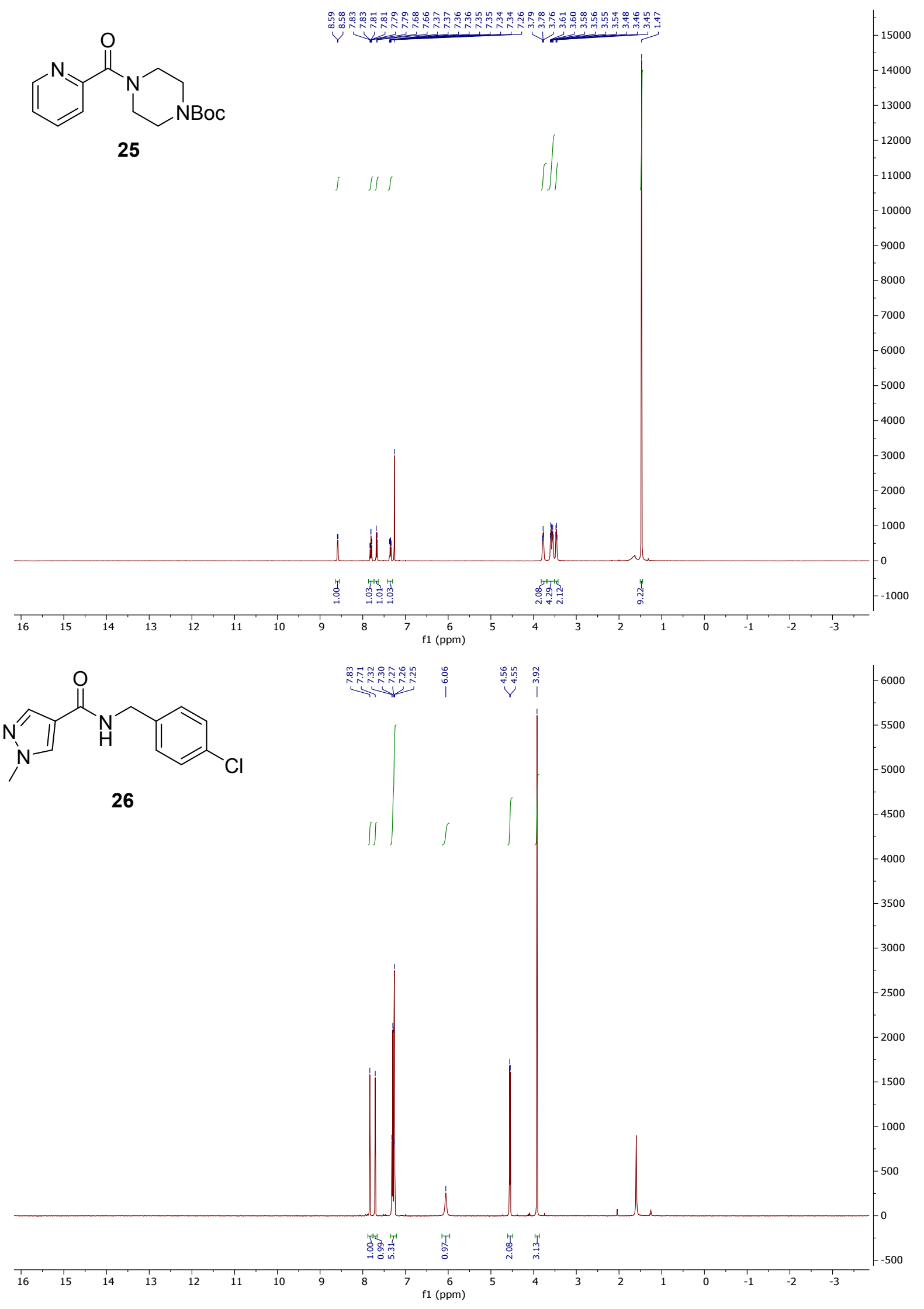




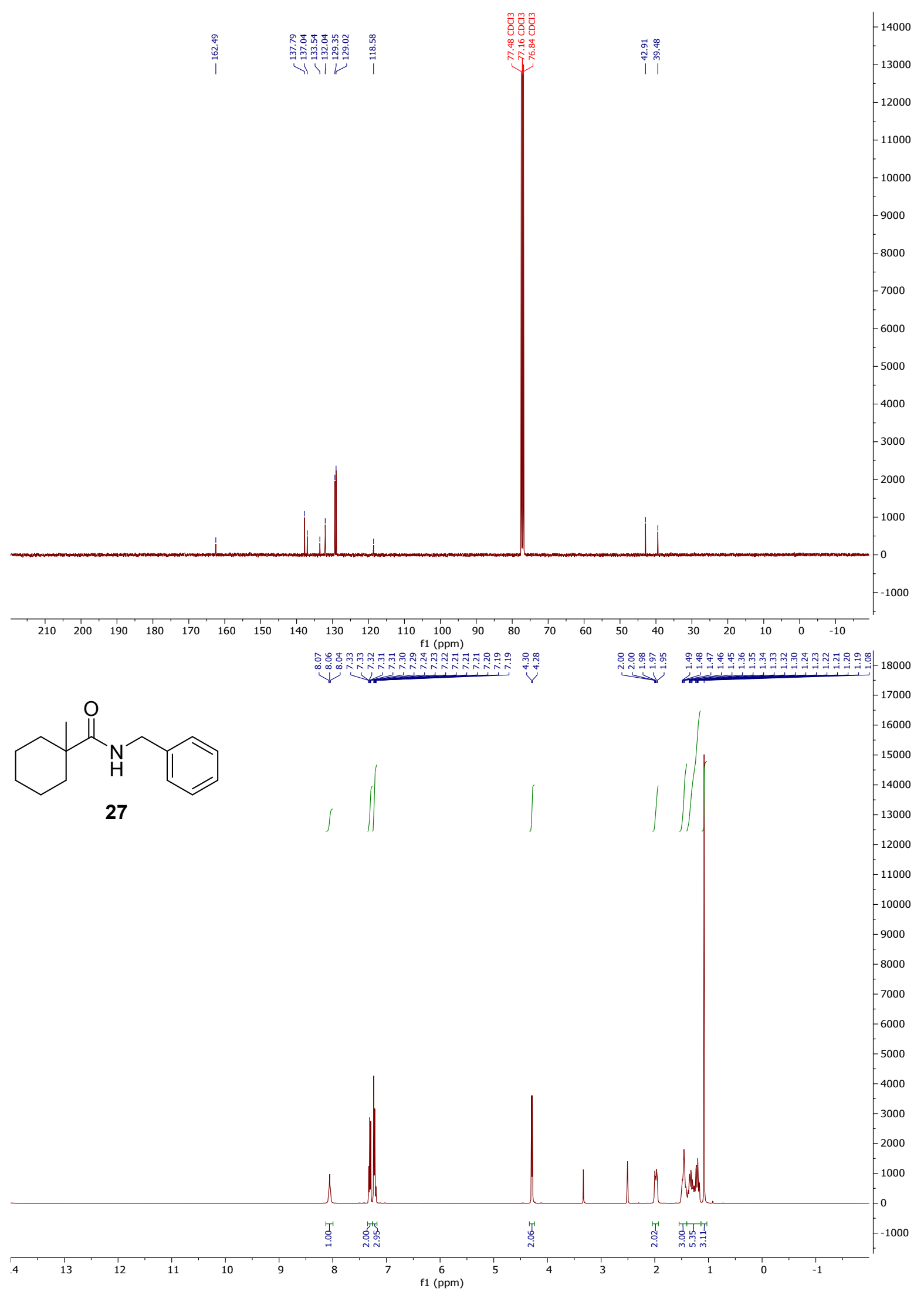




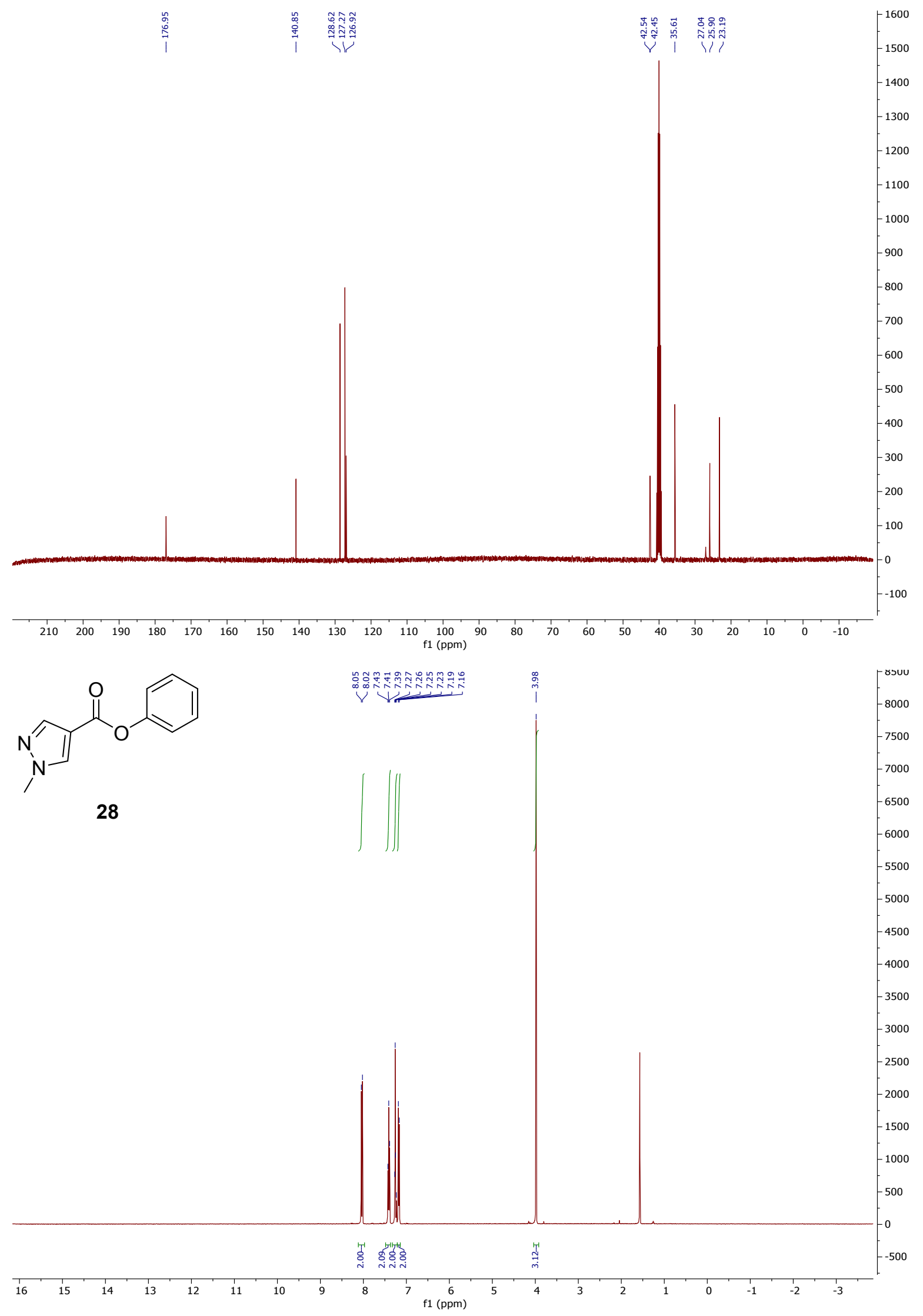


<smiles>CC1(C(=O)OCc2ccccc2)CCCCC1</smiles>

29
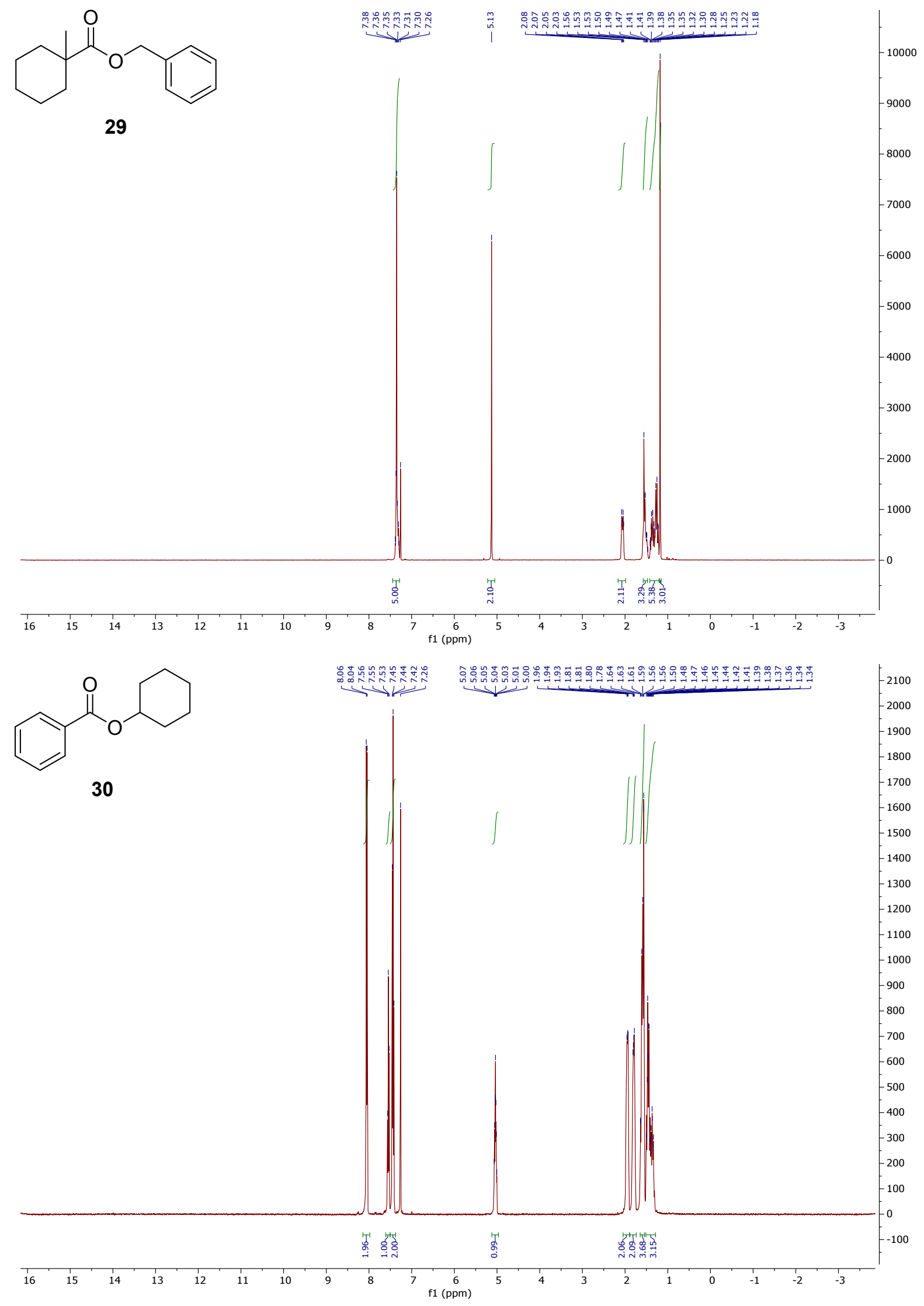

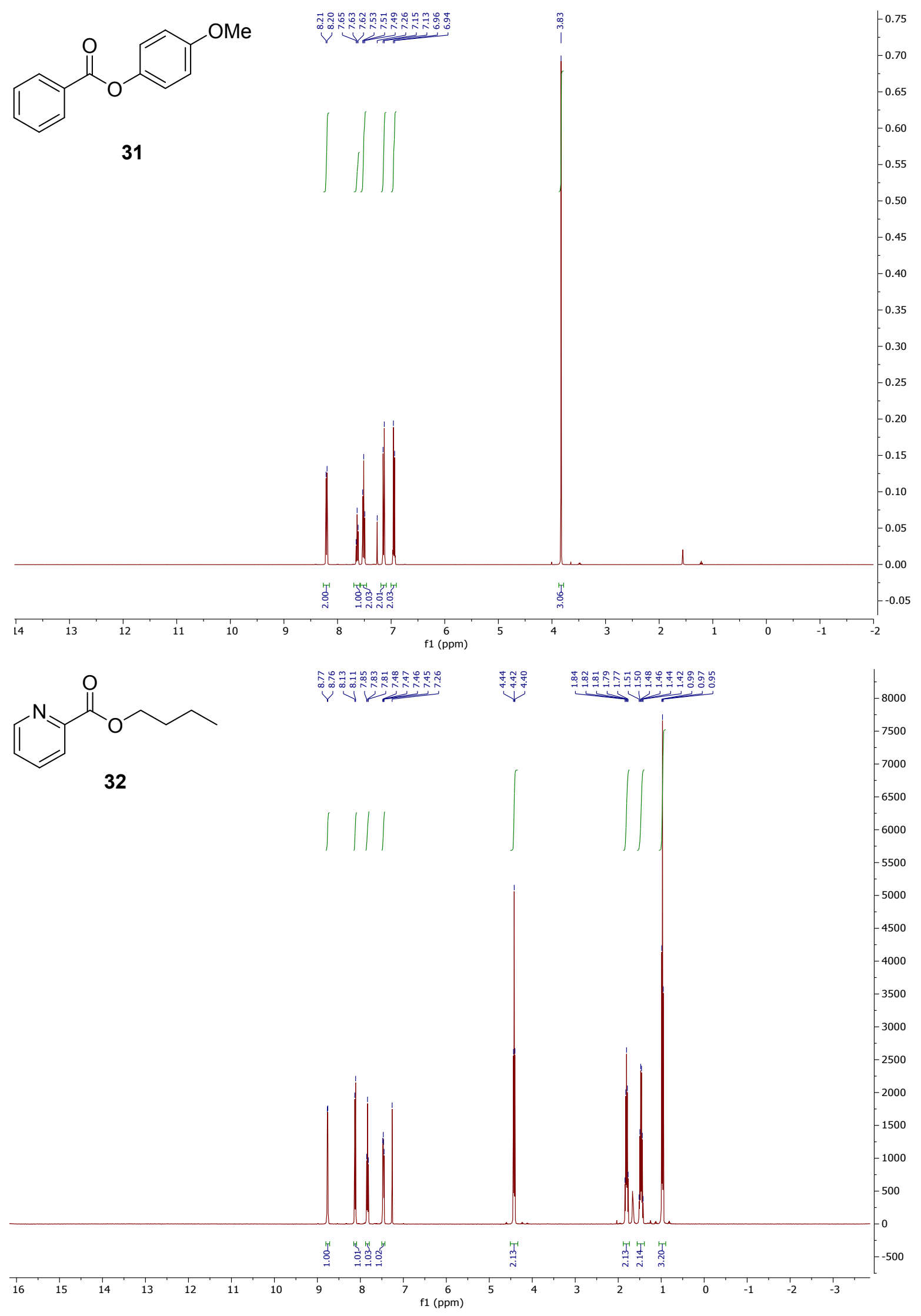

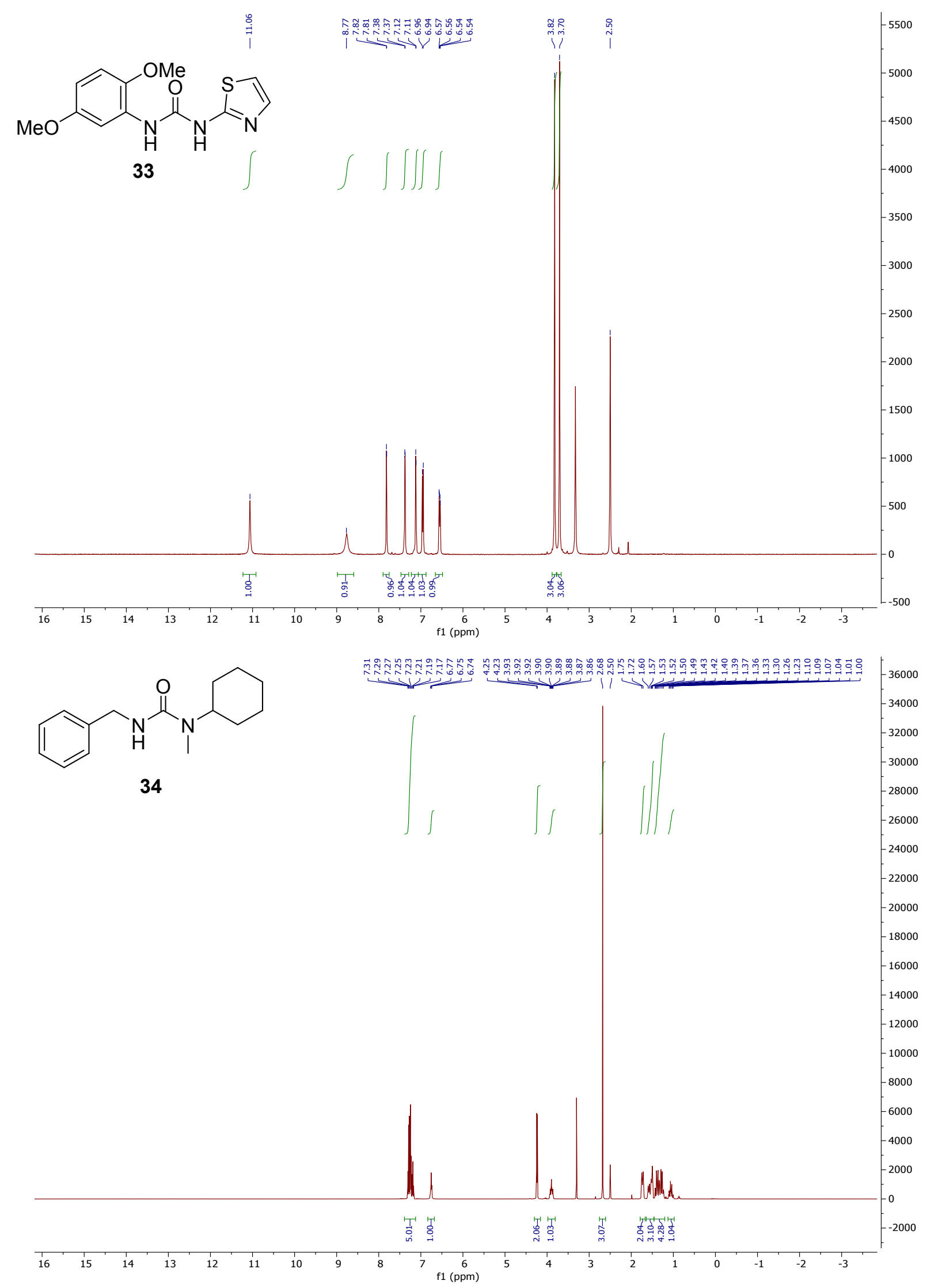


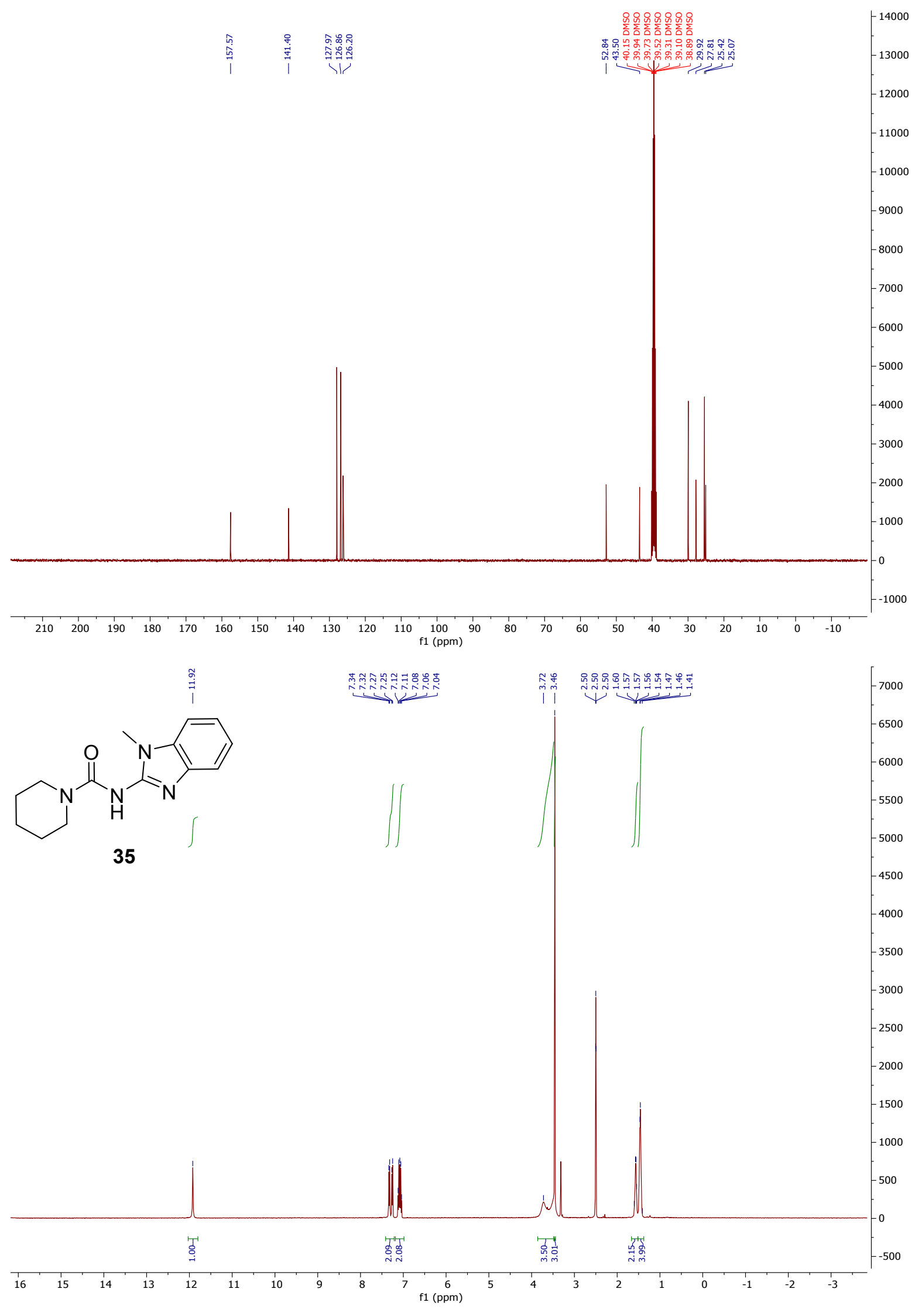




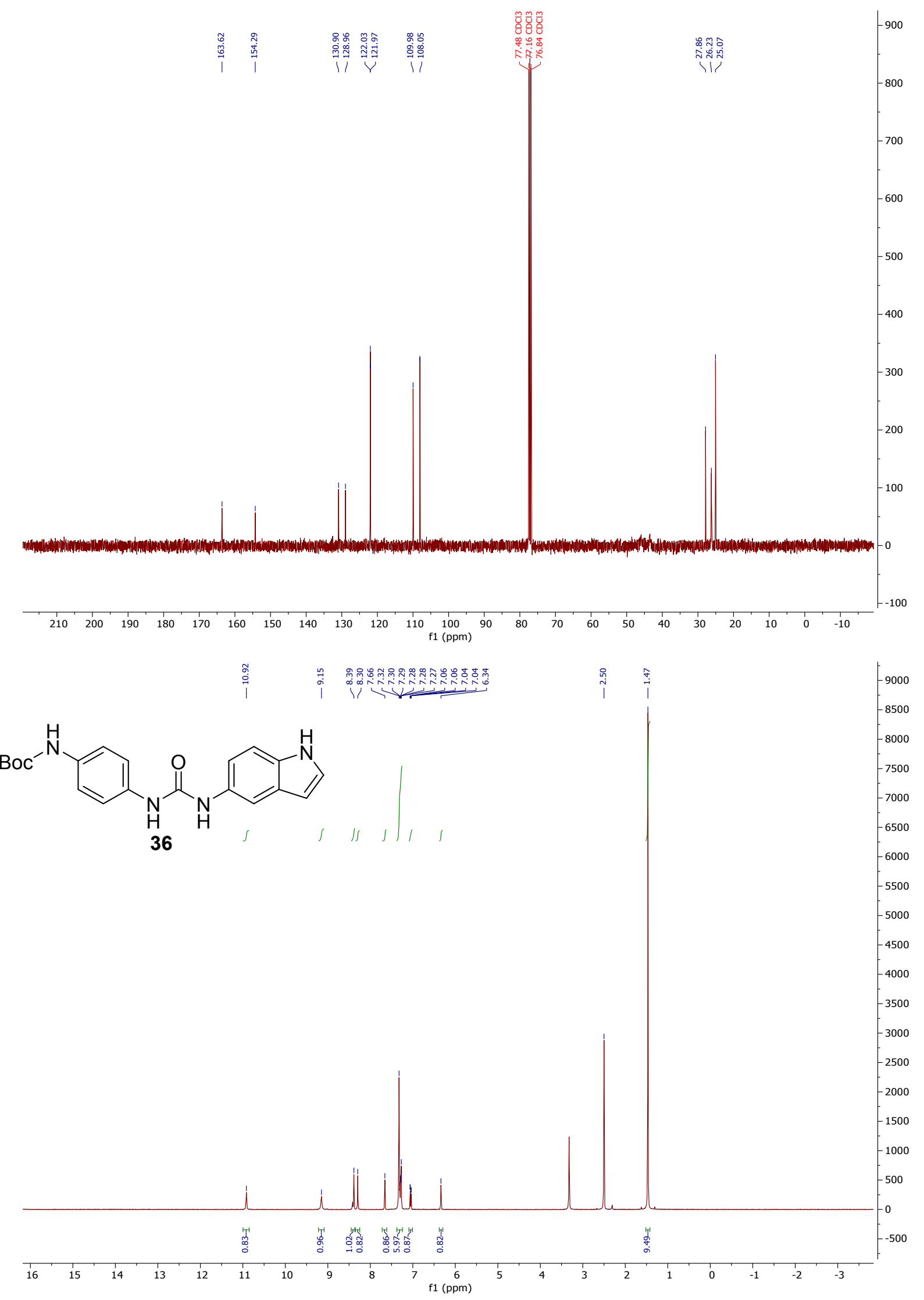




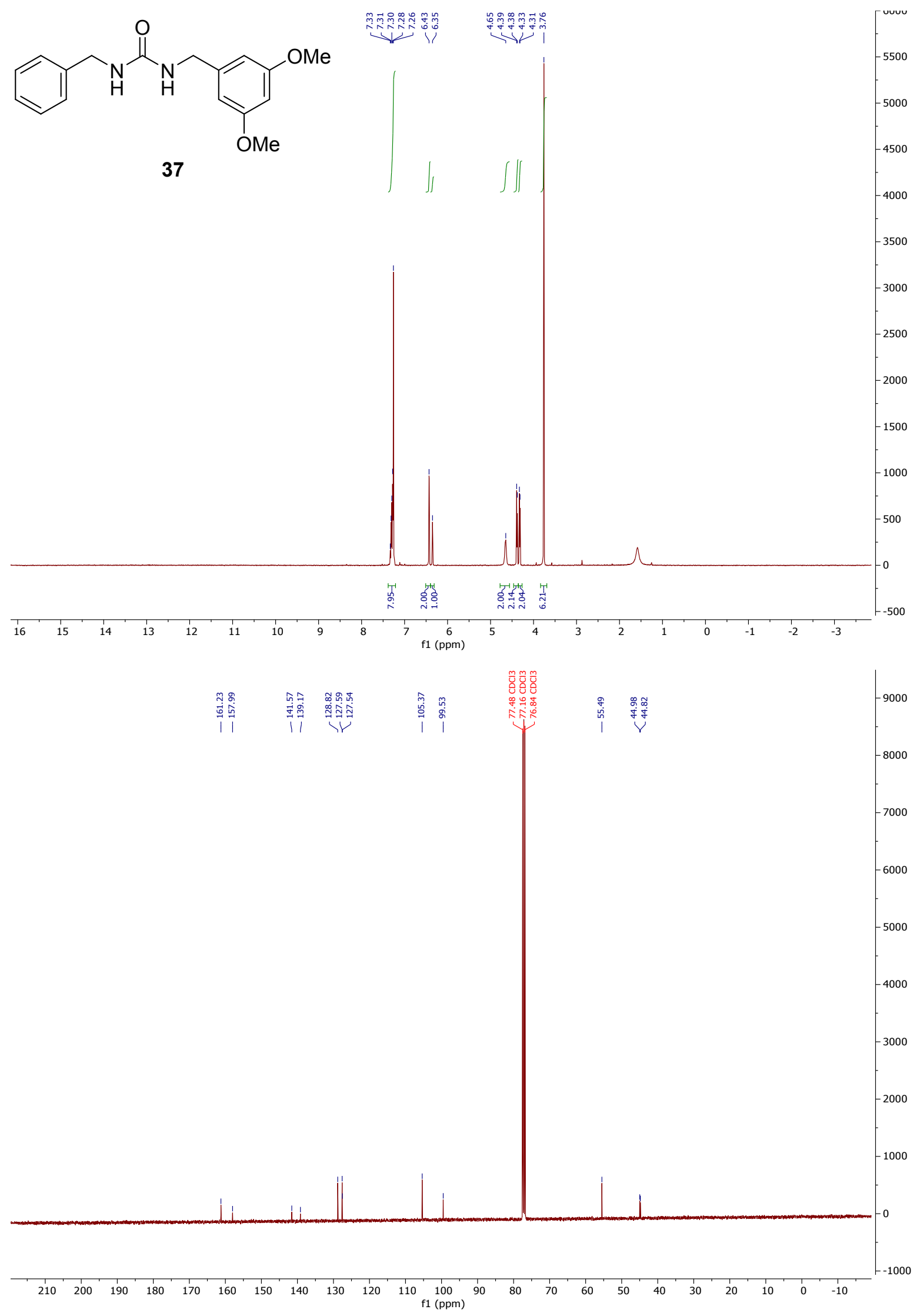




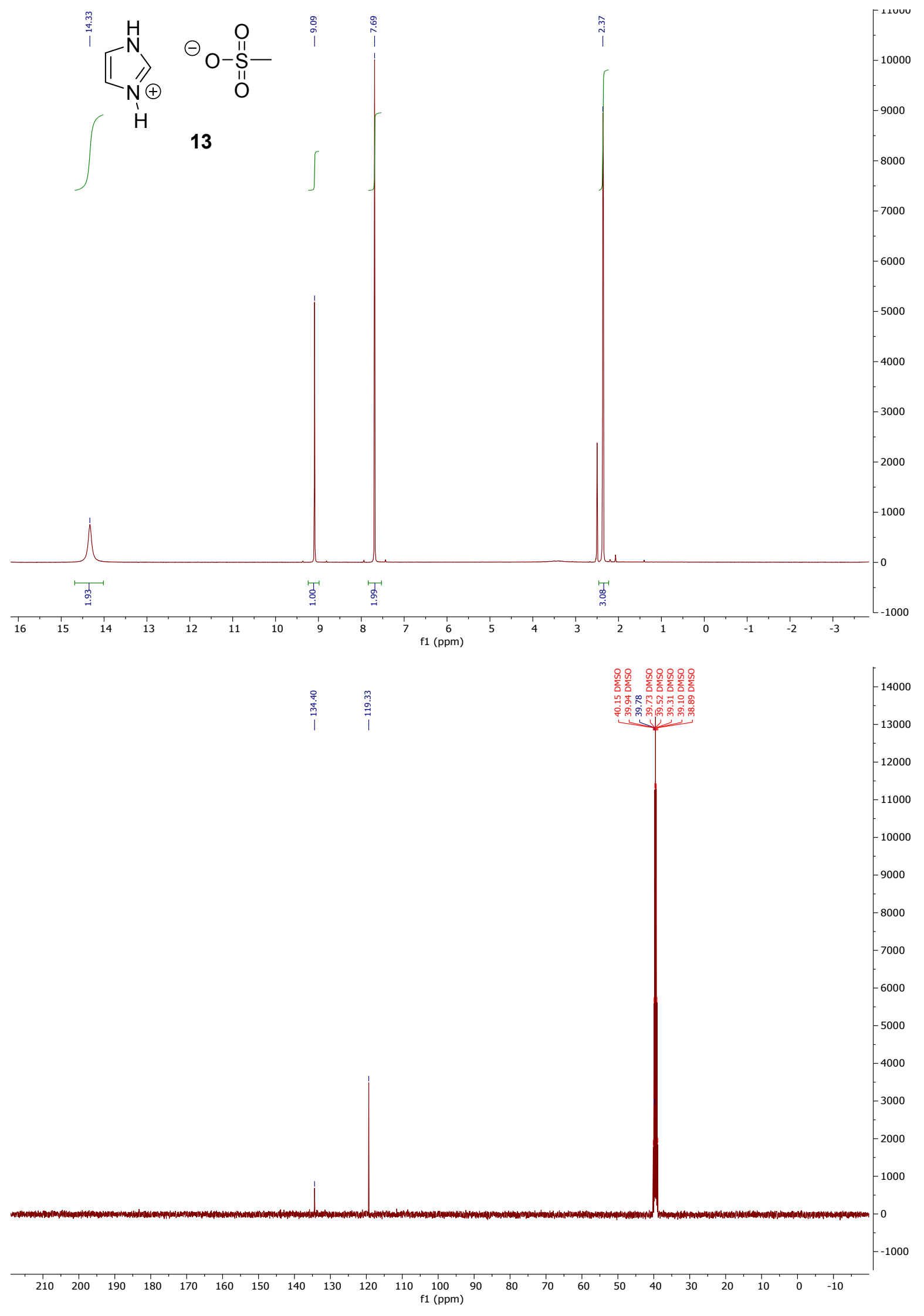


${ }^{1}$ Meng, G.; Lei, P.; Szostak, M. A General Method for Two-Step Transamidation of Secondary Amides Using Commercially Available, Air- and Moisture-Stable Palladium/NHC ( $\mathrm{N}$ Heterocyclic Carbene) Complexes. Org. Lett. 2017, 19, 2158-2161.

${ }^{2}$ Akbar, A.; McNeil, N. M. R.; Albert, M. R.; Ta, V.; Adhikary, G.; Bourgeois, K.; Eckert, R. L.; Keillor, J. W. Structure-Activity Relationships of Potent, Targeted Covalent Inhibitors That Abolish Both the Transamidation and GTP Binding Activities of Human Tissue Transglutaminase. J. Med. Chem. 2017, 60, 7910-7927.

${ }^{3}$ Wang, D.; Ge, C.; Yu, X.; Wan, H.; Xu, X. Transition-Metal-Free Direct Arylation and Esterification Reaction of Unprotected Indolylcarboxylic Acid Derivatives: A New Entry to 2(1H-Indol-2-yl)-5-(phenylthio)-1,3,4-oxadiazoles and Aryl $1 H$-Indole-2-carboxylates. Synlett 2016, 27, 2616-2620.

${ }^{4}$ Hayashi, Y.; Li, J.; Asano, H.; Sakamoto, D. Sterically Congested Ester Formation from $\alpha-$ Substituted Malononitrile and Alcohol by an Oxidative Method Using Molecular Oxygen. Eur. J. Org. Chem. 2019, 4, 675-677.

${ }^{5}$ Nishio, Y.; Kawazu, A.; Hirano, S.; Matsubara H. Preparation of fluorous Yamaguchi reagents and evaluation of their reactivity in esterification. Tetrahedron 2016, 72, 720-725.

${ }^{6}$ Liu, H.-X.; Dang, Y.-Q.; Yuan, Y.-F.; Xu, Z.-F.; Qiu, S.-X.; Tan, H.-B. Diacyl Disulfide: A Reagent for Chemoselective Acylation of Phenols Enabled by 4-( $N, N$-Dimethylamino)pyridine Catalysis. Org. Lett. 2016, 18, 5584-5587.

${ }^{7}$ Wang, L.; Neumann, H.; Spannenberg, A.; Beller, M. Practical in situ-generation of phosphinite ligands for palladium-catalyzed carbonylation of (hetero)aryl bromides forming esters. Chem. Commun. 2017, 53, 7469-7472.

${ }^{8}$ Vinogradova, E. V.; Fors, B. P.; Buchwald, S. L. Palladium-Catalyzed Cross-Coupling of Aryl Chlorides and Triflates with Sodium Cyanate: A Practical Synthesis of Unsymmetrical Ureas. $J$. Am. Chem. Soc. 2012, 134, 11132-11135. 